\title{
Phenomenology of the Higgs effective Lagrangian via
} FEYNRuLES

\author{
Adam Alloul, ${ }^{a}$ Benjamin Fuks ${ }^{b, c}$ and Verónica Sanz ${ }^{d}$ \\ ${ }^{a}$ Groupe de Recherche de Physique des Hautes Énergies (GRPHE), Université de Haute-Alsace, \\ IUT Colmar, 34 rue du Grillenbreit BP 50568, 68008 Colmar Cedex, France \\ ${ }^{b}$ Theory Division, Physics Department, CERN, \\ CH-1211 Geneva 23, Switzerland \\ ${ }^{c}$ Institut Pluridisciplinaire Hubert Curien/Département Recherches Subatomiques, \\ Université de Strasbourg/CNRS-IN2P3, 23 rue du Loess, F-67037 Strasbourg, France \\ ${ }^{d}$ Department of Physics and Astronomy, University of Sussex, \\ Brighton BN1 9QH, U.K. \\ E-mail: adam.alloul@iphc.cnrs.fr, benjamin.fuks@cern.ch, \\ v.sanz@sussex.ac.uk
}

ABstract: The Higgs discovery and the lack of any other hint for new physics favor a description of non-standard Higgs physics in terms of an effective field theory. We present an implementation of a general Higgs effective Lagrangian containing operators up to dimension six in the framework of FEYNRULES and provide details on the translation between the mass and interaction bases, in particular for three- and four-point interaction vertices involving Higgs and gauge bosons. We illustrate the strengths of this implementation by using the UFO interface of FEYNRULES capable to generate model files that can be understood by the MADGRAPH 5 event generator and that have the specificity to contain all interaction vertices, without any restriction on the number of external legs or on the complexity of the Lorentz structures. We then investigate several new physics effects in total rates and differential distributions for different Higgs production modes, including gluon fusion, associated production with a gauge boson and di-Higgs production. We finally study contact interactions of gauge and Higgs bosons to fermions.

Keywords: Phenomenological Models, Monte Carlo Simulations

ARXIV EPRINT: 1310.5150 


\section{Contents}

1 Introduction 1

2 Effective Lagrangians for a Higgs doublet $\quad 3$

2.1 The most general effective Higgs Lagrangian in the gauge eigenbasis 3

2.2 The most general effective Higgs Lagrangian in the mass eigenbasis $\quad 7$

3 Experimental constraints on dimension-six effective operators $\quad 14$

4 Phenomenological examples $\quad \mathbf{1 5}$

4.1 Interactions between the Higgs boson and the electroweak gauge bosons 15

$\begin{array}{lll}\text { 4.1.1 Probing the custodial symmetry } & 16\end{array}$

4.1.2 Kinematics of the four-lepton system issued from a $h \rightarrow Z^{*} Z^{(*)}$ decay 17

4.1.3 Kinematics of the two-lepton system issued from a $h \rightarrow W^{*} W^{(*)}$ decay 18

4.1.4 Correlations of new physics effects in the Higgs boson partial widths 19

4.2 Higgs boson production in association with a vector boson 21

4.2.1 New physics effects in total cross sections for associated Higgs and gauge boson production

4.2.2 Invariant mass of a two-body system constituted of a Higgs boson and a gauge boson

4.3 Di-Higgs production in vector boson fusion

4.4 Associated production of a Higgs and gauge boson from contact interactions with fermions

\section{Introduction}

The discovery of a new state featuring the characteristics of a Higgs boson [1,2] has led to an intense effort to determine whether it consists of the Standard Model (SM) Higgs boson $[3,4]$. On the one hand, its quantum numbers and properties have been theoretically studied in the context of the Standard Model and many of its extensions [5-46], while on the other hand, both Tevatron and LHC experiments have determined that the resonance is likely a scalar particle with properties close to those expected from a Standard Model Higgs boson [47-49]. In particular, dedicated studies have tried to unravel new physics by investigating possible deviations of the couplings of the new state to the Standard Model particles [50-79].

Alas, no direct evidence of physics beyond the Standard Model has been observed in the light of current data [80, 81], up to some anomalous multilepton events recorded by the CMS 
detector [82] which could find a possible explanation from gauge-mediated supersymmetrybreaking models [83]. With this state of affairs, a practical way for investigating new physics lies in a description based on an effective field theory valid up to a scale $\Lambda$ lying around the $\mathrm{TeV}$ scale. In this context, the dynamics of the elementary particles is described through higher-dimensional operators featuring the Standard Model fields and only constrained by the SM symmetry group. This approach allows to characterize the properties of the newly observed Higgs boson, and also has the advantages to be renormalizable order by order in the $E / \Lambda$ expansion, $E$ being a typical energy scale for the processes of interest, as well as to exhibit a small number of free parameters as the gauge symmetries and the hierarchy among the operators of different canonical dimensions strongly constrain the set of operators relevant for a given purpose. Consequently, the path of effective field theories for Higgs physics has been widely explored [84-99], although one of its major drawbacks consists of the loss of unitarity, and thus of predictivity, for energy scales greater than $\Lambda$.

In this work, we focus on the dominant dimension-six operators related to Higgs physics, i.e., those involving at least one Higgs or gauge field, and neglect possible fourfermion interactions allowed by the SM gauge symmetries. Whilst limits on the magnitude of the associated Wilson coefficients can be extracted from LEP and Tevatron data, Higgs signal strengths deduced from measurements by both the ATLAS and CMS collaborations also now imply constraints on a subset of the allowed operators [100-105]. However, as more information on the Higgs-boson properties is obtained by LHC experiments, investigating new physics effects with a simple fit of the Higgs signal strengths clearly becomes too naive. More statistics indeed allows one for employing various kinematical distributions as powerful handles to look for physics beyond the Standard Model in the Higgs sector. In these perspectives, it is necessary to rely on sophisticated tools that cover the implementation of the Higgs effective operators in Monte Carlo simulation programs, the latter further leading to a possible recasting of the experimental analyses and a subsequent comparison of the theoretical predictions, in the framework of an effective field theory for Higgs physics, with experimental data.

Within the last few years, a framework based on the FeYnRules package [106113] has been developed in order to facilitate the implementation (and validation) of any beyond the Standard Model theory in multi-purpose matrix-element generators [114]. In particular, its virtues have been illustrated in the context of the CALCHEP [115118], Feynarts/FormCalc [119-124], MadGraph [125-129], Sherpa [130, 131] and WhizARD $[132,133]$ programs. Furthermore, it also offers the possibility to translate any particle physics model in terms of a PүтноN library under the so-called Universal FeynRules Output (UFO) format [134]. Previously designed model formats for Monte Carlo programs are usually imposing restrictions on the color and/or Lorentz structures and the number of external legs that are allowed in the interaction vertices so that the UFO has been conceived to overcome such limitations. It is therefore suitable for effective field theories that often contain vertices with uncommon Lorentz structures.

We present in this work an implementation in FEYNRULES of a Higgs Effective Field Theory that includes a set of independent dimension-six operators assumed to encompass all possible effects of new physics on the Higgs sector. This set of operators can further be easily 
complemented by 22 four-fermion operators, irrelevant for Higgs physics (at least at leadingorder and in the context of the LHC phenomenology) and thus omitted here, in order to achieve a full basis of independent dimension-six operators featuring only Standard Model fields. In this way, high-energy physics programs such as Aloha [135], GoSam [136, 137], Herwig $++[138,139]$, MadAnalysis 5 [140, 141] and MadGraph 5, capable to fully handle a UFO model, can be directly employed for phenomenological investigations of new physics in the context of the effective field theory model presented in this work. For the sake of the examples, we demonstrate the usefulness of such an implementation by studying various beyond the Standard Model effects in both Higgs production rates and kinematical distributions by means of the FEYNRULES and MADGRAPH 5 packages and their interface through the UFO.

Our work is organized as follows. In section 2, we extensively describe the operators which are included in our implementation. Next, we move onto studying several properties of the Higgs boson as examples of phenomenological studies that can be performed from this implementation. Their thorough study, possibly carried at the most sophisticated level of collider physics simulations, is however beyond the scope of this work, as well as a careful design of experimentally allowed values for the Wilson coefficients of the various effective operators that we briefly comment in section 3. In section 4.1, we firstly focus on Higgsboson production by gluon fusion and on the constraints that can be possibly extracted on the custodial symmetry or from measurements of angular distributions in the case the Higgs boson decays via intermediate gauge bosons. Secondly, we consider in section 4.2 the associated production of a Higgs boson with one weak boson and investigate the ratio of the total production rates at center-of-mass energies of $8 \mathrm{TeV}$ and $14 \mathrm{TeV}$ as well as the invariant-mass spectrum of the Higgs and vector boson system. Thirdly, we assess the effects of some effective operators on the production of a Higgs-boson pair in section 4.3, focusing on boosted topologies. Finally, we study in section 4.4 contact interactions of a single Higgs field, a single gauge boson and a fermion pair and compare the LHC reach to limits extracted from LEP data. Our conclusions are given in section 5.

\section{Effective Lagrangians for a Higgs doublet}

\subsection{The most general effective Higgs Lagrangian in the gauge eigenbasis}

The Standard Model of particle physics is a quantum field theory which describes the elementary particles and their interactions based on the $\mathrm{SU}(3)_{\mathrm{c}} \times \mathrm{SU}(2)_{\mathrm{L}} \times \mathrm{U}(1)_{\mathrm{Y}}$ gauge symmetry. The particle content of the model can be classified in terms of a gauge sector, consisting of vector fields responsible for the mediation of the interactions, a chiral sector with the matter building blocks and eventually a Higgs sector related to the breaking of the electroweak symmetry. We now introduce our notations and start with the gauge vector fields lying in the adjoint representation of the relevant gauge subgroup,

$$
\mathrm{SU}(3)_{\mathrm{c}} \rightarrow \mathrm{G}_{\mu}^{\mathrm{a}}=(\underset{\sim}{\mathbf{8}} \underset{\sim}{\mathbf{1}}, 0), \quad \mathrm{SU}(2)_{\mathrm{L}} \rightarrow \mathrm{W}_{\mu}^{\mathrm{k}}=(\underset{\sim}{\mathbf{1}}, \underset{\sim}{\mathbf{3}}, 0), \quad \mathrm{U}(1)_{\mathrm{Y}} \rightarrow \mathrm{B}_{\mu}=(\underset{\sim}{\mathbf{1}}, \underset{\sim}{\mathbf{1}}, 0),
$$


which we show together with their full representation under the Standard Model gauge group and explicitly indicate the adjoint gauge indices. The chiral content of the theory is defined by three generations of left-handed and right-handed quark $\left(Q_{L}, u_{R}\right.$ and $\left.d_{R}\right)$ and lepton $\left(L_{L}\right.$ and $\left.e_{R}\right)$ fields,

$$
\begin{aligned}
& Q_{L}=\left(\begin{array}{c}
u_{L} \\
d_{L}
\end{array}\right)=\left(\underset{\sim}{\mathbf{3}} \underset{\sim}{\mathbf{2}}, \frac{1}{6}\right), \quad u_{R}=\left(\underset{\sim}{\mathbf{3}}, \underset{\sim}{\mathbf{1}}, \frac{2}{3}\right), \quad d_{R}=\left(\underset{\sim}{\mathbf{3}}, \underset{\sim}{\mathbf{1}},-\frac{1}{3}\right), \\
& L_{L}=\left(\begin{array}{c}
\nu_{L} \\
\ell_{L}
\end{array}\right)=\left(\underset{\sim}{\mathbf{1}} \underset{\sim}{\mathbf{2}},-\frac{1}{2}\right), \quad e_{R}=(\underset{\sim}{\mathbf{1}}, \underset{\sim}{\mathbf{1}},-1),
\end{aligned}
$$

which we again present together with their representation under the Standard Model gauge group. Finally, the Higgs sector contains a single $\mathrm{SU}(2)_{\mathrm{L}}$ doublet of fields,

$$
\Phi=\left(\begin{array}{c}
-i G^{+} \\
\frac{1}{\sqrt{2}}\left[v+h+i G^{0}\right]
\end{array}\right)=\left(\underset{\sim}{\mathbf{1}}, \underset{\sim}{\mathbf{2}}, \frac{1}{2}\right) .
$$

With the first equality, we show the component fields of the doublet after shifting the neutral field $h$ by its vacuum expectation value $v$. Moreover, we have included the Goldstone bosons $G^{+, 0}$ to be eaten by the weak boson to get their longitudinal degree of freedom.

In the effective field theory-based approach that we adopt, the usual Standard Model Lagrangian $\mathcal{L}_{\mathrm{SM}}$ is supplemented by higher-dimensional operators that parametrize the possible effects of non-observed states assumed to appear at energies larger than an effective scale identified with the $W$-boson mass $m_{W}$ or equivalently with the vacuum expectation value of the Higgs field $v$. Restricting ourselves to operators of dimension less than or equal to six, the most general gauge-invariant Lagrangian $\mathcal{L}$ is known for a long time [84-86] and can be expressed, in a convenient basis of independent operators $\mathcal{O}_{i}[93,94]$, as

$$
\mathcal{L}=\mathcal{L}_{\mathrm{SM}}+\sum_{i} \bar{c}_{i} \mathcal{O}_{i}=\mathcal{L}_{\mathrm{SM}}+\mathcal{L}_{\mathrm{SILH}}+\mathcal{L}_{C P}+\mathcal{L}_{F_{1}}+\mathcal{L}_{F_{2}}+\mathcal{L}_{G}
$$

assuming baryon and lepton number conservation. Moreover, we adopt the decomposition of ref. [97] and normalize the Wilson coefficients $\bar{c}_{i}$ as such. Other choices for the operator basis yield different physics interpretations and care must be taken when comparing various works on the topic. According to the purposes, other bases might be more adequate.

The first piece of this Lagrangian, $\mathcal{L}_{\mathrm{SILH}}$, corresponds to the a popular set of $C P$ conserving operators involving the Higgs doublet $\Phi$. Their (conventional) normalization is inspired by scenarios where the Higgs field is part of a strongly interacting sector,

$$
\begin{aligned}
\mathcal{L}_{\mathrm{SILH}}= & \frac{\bar{c}_{H}}{2 v^{2}} \partial^{\mu}\left[\Phi^{\dagger} \Phi\right] \partial_{\mu}\left[\Phi^{\dagger} \Phi\right]+\frac{\bar{c}_{T}}{2 v^{2}}\left[\Phi^{\dagger} \overleftrightarrow{D}^{\mu} \Phi\right]\left[\Phi^{\dagger} \overleftrightarrow{D}_{\mu} \Phi\right]-\frac{\bar{c}_{6} \lambda}{v^{2}}\left[\Phi^{\dagger} \Phi\right]^{3} \\
& -\left[\frac{\bar{c}_{u}}{v^{2}} y_{u} \Phi^{\dagger} \Phi \Phi^{\dagger} \cdot \bar{Q}_{L} u_{R}+\frac{\bar{c}_{d}}{v^{2}} y_{d} \Phi^{\dagger} \Phi \Phi \bar{Q}_{L} d_{R}+\frac{\bar{c}_{l}}{v^{2}} y_{\ell} \Phi^{\dagger} \Phi \Phi \bar{L}_{L} e_{R}+\text { h.c. }\right] \\
& +\frac{i g \bar{c}_{W}}{m_{W}^{2}}\left[\Phi^{\dagger} T_{2 k} \overleftrightarrow{D}^{\mu} \Phi\right] D^{\nu} W_{\mu \nu}^{k}+\frac{i g^{\prime} \bar{c}_{B}}{2 m_{W}^{2}}\left[\Phi^{\dagger} \overleftrightarrow{D^{\mu}} \Phi\right] \partial^{\nu} B_{\mu \nu} \\
& +\frac{2 i g \bar{c}_{H W}}{m_{W}^{2}}\left[D^{\mu} \Phi^{\dagger} T_{2 k} D^{\nu} \Phi\right] W_{\mu \nu}^{k}+\frac{i g^{\prime} \bar{c}_{H B}}{m_{W}^{2}}\left[D^{\mu} \Phi^{\dagger} D^{\nu} \Phi\right] B_{\mu \nu} \\
& +\frac{g^{\prime 2} \bar{c}_{\gamma}}{m_{W}^{2}} \Phi^{\dagger} \Phi B_{\mu \nu} B^{\mu \nu}+\frac{g_{s}^{2} \bar{c}_{g}}{m_{W}^{2}} \Phi^{\dagger} \Phi G_{\mu \nu}^{a} G_{a}^{\mu \nu},
\end{aligned}
$$


where the Wilson coefficients $\bar{c}$ are free parameters, $\lambda$ stands for the Higgs quartic coupling and $y_{u}, y_{d}$ and $y_{\ell}$ are the $3 \times 3$ Yukawa coupling matrices in flavor space (all flavor indices are understood for clarity). In this expression, we also denote the $\mathrm{U}(1)_{\mathrm{Y}}, \mathrm{SU}(2)_{\mathrm{L}}$ and $\mathrm{SU}(3)_{\mathrm{c}}$ coupling constants by $g^{\prime}, g$ and $g_{s}$, whereas the generators of $\mathrm{SU}(2)$ in the fundamental representation are given by $T_{2 k}=\sigma_{k} / 2, \sigma_{k}$ being the Pauli matrices. Additionally, we have introduced the Hermitian derivative operators $\stackrel{\vec{D}}{\mu}_{\mu}$ defined as

$$
\Phi^{\dagger} \overleftrightarrow{D}_{\mu} \Phi=\Phi^{\dagger} D^{\mu} \Phi-D_{\mu} \Phi^{\dagger} \Phi
$$

and the $\mathrm{SU}(2)$ invariant products

$$
Q_{L} \cdot \Phi=\epsilon_{i j} Q_{L}^{i} \Phi^{j} \quad \text { and } \quad \Phi^{\dagger} \cdot \bar{Q}_{L}=\epsilon^{i j} \Phi_{i}^{\dagger} \bar{Q}_{L j},
$$

the rank-two antisymmetric tensors being defined by $\epsilon_{12}=1$ and $\epsilon^{12}=-1$. Finally, our conventions for the gauge-covariant derivatives and the gauge field strength tensors are

$$
\begin{aligned}
B_{\mu \nu}= & \partial_{\mu} B_{\nu}-\partial_{\nu} B_{\mu}, \\
W_{\mu \nu}^{k}= & \partial_{\mu} W_{\nu}^{k}-\partial_{\nu} W_{\mu}^{k}+g \epsilon_{i j}{ }^{k} W_{\mu}^{i} W_{\nu}^{j}, \\
G_{\mu \nu}^{a}= & \partial_{\mu} G_{\nu}^{a}-\partial_{\nu} G_{\mu}^{a}+g_{s} f_{b c}{ }^{a} G_{\mu}^{b} G_{\nu}^{c}, \\
D_{\rho} W_{\mu \nu}^{k}= & \partial_{\mu} \partial_{\rho} W_{\nu}^{k}-\partial_{\nu} \partial_{\rho} W_{\mu}^{k}+g \epsilon_{i j}{ }^{k} \partial_{\rho}\left[W_{\mu}^{i} W_{\nu}^{j}\right]+g \epsilon_{i j}{ }^{k} W_{\rho}^{i}\left[\partial_{\mu} W_{\nu}^{j}-\partial_{\nu} W_{\mu}^{j}\right] \\
& \quad+g^{2} W_{\rho i}\left[W_{\nu}^{i} W_{\mu}^{k}-W_{\mu}^{i} W_{\nu}^{k}\right], \\
D_{\mu} \Phi= & \partial_{\mu} \Phi-\frac{1}{2} i g^{\prime} B_{\mu} \Phi-i g T_{2 k} W_{\mu}^{k} \Phi,
\end{aligned}
$$

$\epsilon_{i j}{ }^{k}$ and $f_{a b}{ }^{c}$ being the structure constants of SU(2) and SU(3). The Lagrangian of eq. (2.5) can be supplemented by extra $C P$-violating operators,

$$
\begin{aligned}
\mathcal{L}_{C P}= & \frac{i g \tilde{c}_{H W}}{m_{W}^{2}} D^{\mu} \Phi^{\dagger} T_{2 k} D^{\nu} \Phi \widetilde{W}_{\mu \nu}^{k}+\frac{i g^{\prime} \tilde{c}_{H B}}{m_{W}^{2}} D^{\mu} \Phi^{\dagger} D^{\nu} \Phi \widetilde{B}_{\mu \nu}+\frac{g^{\prime 2} \tilde{c}_{\gamma}}{m_{W}^{2}} \Phi^{\dagger} \Phi B_{\mu \nu} \widetilde{B}^{\mu \nu} \\
& +\frac{g_{s}^{2} \tilde{c}_{g}}{m_{W}^{2}} \Phi^{\dagger} \Phi G_{\mu \nu}^{a} \widetilde{G}_{a}^{\mu \nu}+\frac{g^{3} \tilde{c}_{3 W}}{m_{W}^{2}} \epsilon_{i j k} W_{\mu \nu}^{i} W_{\rho}^{\nu j} \widetilde{W}^{\rho \mu k}+\frac{g_{s}^{3} \tilde{c}_{3 G}}{m_{W}^{2}} f_{a b c} G_{\mu \nu}^{a} G_{\rho}^{\nu b} \widetilde{G}^{\rho \mu c},
\end{aligned}
$$

where the dual field strength tensors are defined by

$$
\widetilde{B}_{\mu \nu}=\frac{1}{2} \epsilon_{\mu \nu \rho \sigma} B^{\rho \sigma}, \quad \widetilde{W}_{\mu \nu}^{k}=\frac{1}{2} \epsilon_{\mu \nu \rho \sigma} W^{\rho \sigma k}, \quad \widetilde{G}_{\mu \nu}^{a}=\frac{1}{2} \epsilon_{\mu \nu \rho \sigma} G^{\rho \sigma a} .
$$

The third term in eq. (2.4) contains interactions between two Higgs fields and a pair of quarks or leptons,

$$
\begin{aligned}
\mathcal{L}_{F_{1}}= & \frac{i \bar{c}_{H Q}}{v^{2}}\left[\bar{Q}_{L} \gamma^{\mu} Q_{L}\right]\left[\Phi^{\dagger} \overleftrightarrow{D}_{\mu} \Phi\right]+\frac{4 i \bar{c}_{H Q}^{\prime}}{v^{2}}\left[\bar{Q}_{L} \gamma^{\mu} T_{2 k} Q_{L}\right]\left[\Phi^{\dagger} T_{2}^{k} \overleftrightarrow{D}_{\mu} \Phi\right] \\
& +\frac{i \bar{c}_{H u}}{v^{2}}\left[\bar{u}_{R} \gamma^{\mu} u_{R}\right]\left[\Phi^{\dagger} \overleftrightarrow{D}_{\mu} \Phi\right]+\frac{i \bar{c}_{H d}}{v^{2}}\left[\bar{d}_{R} \gamma^{\mu} d_{R}\right]\left[\Phi^{\dagger} \overleftrightarrow{D}_{\mu} \Phi\right] \\
& -\left[\frac{i \bar{c}_{H u d}}{v^{2}}\left[\bar{u}_{R} \gamma^{\mu} d_{R}\right]\left[\Phi \cdot \overleftrightarrow{D}{ }_{\mu} \Phi\right]+\text { h.c. }\right] \\
& +\frac{i \bar{c}_{H L}}{v^{2}}\left[\bar{L}_{L} \gamma^{\mu} L_{L}\right]\left[\Phi^{\dagger} \overleftrightarrow{D}_{\mu} \Phi\right]+\frac{4 i \bar{c}_{H L}^{\prime}}{v^{2}}\left[\bar{L}_{L} \gamma^{\mu} T_{2 k} L_{L}\right]\left[\Phi^{\dagger} T_{2}^{k} \overleftrightarrow{D}_{\mu} \Phi\right] \\
& +\frac{i \bar{c}_{H e}}{v^{2}}\left[\bar{e}_{R} \gamma^{\mu} e_{R}\right]\left[\Phi^{\dagger} \overleftrightarrow{D}_{\mu} \Phi\right],
\end{aligned}
$$


whilst the fourth term of this Lagrangian addresses the interactions of a quark or lepton pair and one single Higgs field and a gauge boson,

$$
\begin{aligned}
\mathcal{L}_{F_{2}}=[ & -\frac{2 g^{\prime} \bar{c}_{u B}}{m_{W}^{2}} y_{u} \Phi^{\dagger} \cdot \bar{Q}_{L} \gamma^{\mu \nu} u_{R} B_{\mu \nu}-\frac{4 g \bar{c}_{u W}}{m_{W}^{2}} y_{u} \Phi^{\dagger} \cdot\left(\bar{Q}_{L} T_{2 k}\right) \gamma^{\mu \nu} u_{R} W_{\mu \nu}^{k} \\
& -\frac{4 g_{s} \bar{c}_{u G}}{m_{W}^{2}} y_{u} \Phi^{\dagger} \cdot \bar{Q}_{L} \gamma^{\mu \nu} T_{a} u_{R} G_{\mu \nu}^{a}+\frac{2 g^{\prime} \bar{c}_{d B}}{m_{W}^{2}} y_{d} \Phi \bar{Q}_{L} \gamma^{\mu \nu} d_{R} B_{\mu \nu} \\
& +\frac{4 g \bar{c}_{d W}}{m_{W}^{2}} y_{d} \Phi\left(\bar{Q}_{L} T_{2 k}\right) \gamma^{\mu \nu} d_{R} W_{\mu \nu}^{k}+\frac{4 g_{s} \bar{c}_{d G}}{m_{W}^{2}} y_{d} \Phi \bar{Q}_{L} \gamma^{\mu \nu} T_{a} d_{R} G_{\mu \nu}^{a} \\
& \left.+\frac{2 g^{\prime} \bar{c}_{e B}}{m_{W}^{2}} y_{\ell} \Phi \bar{L}_{L} \gamma^{\mu \nu} e_{R} B_{\mu \nu}+\frac{4 g \bar{c}_{e W}}{m_{W}^{2}} y_{\ell} \Phi\left(\bar{L}_{L} T_{2 k}\right) \gamma^{\mu \nu} e_{R} W_{\mu \nu}^{k}+\text { h.c. }\right] .
\end{aligned}
$$

In this expression, the matrices $T_{a}$ are the generators of the $\mathrm{SU}(3)$ group in the fundamental representation and the $\gamma^{\mu \nu}$ quantities, defined by

$$
\gamma^{\mu \nu}=\frac{i}{4}\left[\gamma^{\mu}, \gamma^{\nu}\right]
$$

are the generators of the Lorentz algebra in the (four-component) spinorial representation. In the most general case, the Wilson coefficients $\bar{c}_{i}$ related to the fermionic operators included in the Lagrangians $\mathcal{L}_{F_{i}}$ are tensors in flavor space and complex quantities.

The last term of eq. (2.4) refers to operators not directly connected to Higgs physics, but that may be important as affecting the gauge sector and possibly modifying the gauge boson self-energies and self-interactions,

$$
\begin{aligned}
\mathcal{L}_{G}= & \frac{g^{3} \bar{c}_{3 W}}{m_{W}^{2}} \epsilon_{i j k} W_{\mu \nu}^{i} W_{\rho}^{\nu j} W^{\rho \mu k}+\frac{g_{s}^{3} \bar{c}_{3 G}}{m_{W}^{2}} f_{a b c} G_{\mu \nu}^{a} G_{\rho}^{\nu b} G^{\rho \mu c}+\frac{\bar{c}_{2 W}}{m_{W}^{2}} D^{\mu} W_{\mu \nu}^{k} D_{\rho} W_{k}^{\rho \nu} \\
& +\frac{\bar{c}_{2 B}}{m_{W}^{2}} \partial^{\mu} B_{\mu \nu} \partial_{\rho} B^{\rho \nu}+\frac{\bar{c}_{2 G}}{m_{W}^{2}} D^{\mu} G_{\mu \nu}^{a} D_{\rho} G_{a}^{\rho \nu}
\end{aligned}
$$

with

$$
\begin{aligned}
D_{\rho} G_{\mu \nu}^{a}= & \partial_{\mu} \partial_{\rho} G_{\nu}^{a}-\partial_{\nu} \partial_{\rho} G_{\mu}^{a}+g_{s} f_{b c}^{a} \partial_{\rho}\left[G_{\mu}^{b} G_{\nu}^{c}\right]+g_{s} f_{b c}^{a} G_{\rho}^{b}\left[\partial_{\mu} G_{\nu}^{c}-\partial_{\nu} G_{\mu}^{c}\right] \\
& +g_{s}^{2} G_{\rho b}\left[G_{\nu}^{b} G_{\mu}^{a}-G_{\mu}^{b} G_{\nu}^{a}\right]
\end{aligned}
$$

recalling that $D_{\mu} W_{k}^{\nu \rho}$ has been defined in eq. (2.8).

Finally, 22 independent baryon and lepton number conserving four-fermion operators are also allowed by gauge invariance. Since they have no effects on Higgs physics, at least at the leading order and in the context of the LHC phenomenology, we omit them from the present manuscript, as already above-mentioned. Moreover, as indicated in ref. [97], two of the 39 operators that have been introduced are redundant and can be removed through

$$
\begin{aligned}
\mathcal{O}_{W} & =-2 \mathcal{O}_{H}+\frac{4}{v^{2}} \Phi^{\dagger} \Phi D^{\mu} \Phi^{\dagger} D_{\mu} \Phi+\mathcal{O}_{H Q}^{\prime}+\mathcal{O}_{H L}^{\prime}, \\
\mathcal{O}_{B} & =2 \tan ^{2} \theta_{W}\left[\sum_{\psi} Y_{\psi} \mathcal{O}_{H \psi}-\mathcal{O}_{T}\right],
\end{aligned}
$$

where we sum over the whole chiral content of the theory and $\theta_{W}$ stands for the weak mixing angle (see eq. (2.17) and eq. (2.18) in section 2.2). We however include them in our 
effective field theory description as according to the specific effect of interest, one choice for an operator basis may be more suitable than another.

The full set of interactions generated by the 39 operators included in the Lagrangian $\mathcal{L}$ of eq. (2.4) have been implemented in a FeynRules $[106,113]$ model file that can be either downloaded from http://feynrules.irmp.ucl.ac.be/wiki/HEL or obtained from the authors upon request. Employing the various interfaces linking FEYNRULEs to Monte Carlo event generators, the effects of those operators can be further studied at colliders. Care must however be taken with the choice of the Monte Carlo generator to employ for this purpose, as most of them have strong requirements on the allowed Lorentz structures for the vertices. ${ }^{1}$ To avoid such limitations and be capable of probing any of the 39 considered operators, we make use, for the few examples of section 4 , of the MADGRAPH 5 program [129] linked to the UFO [134] version of the model presented above.

\subsection{The most general effective Higgs Lagrangian in the mass eigenbasis}

After electroweak symmetry breaking to electromagnetism, i.e., when the Higgs field acquires its vacuum expectation value, the gauge eigenstates introduced in eq. (2.1) mix to the physical massive $W$-boson and $Z$-boson as well as to the photon $A$ which remains massless,

$$
\begin{aligned}
W_{\mu}^{ \pm} & =\frac{1}{\sqrt{2}}\left(W_{\mu}^{1} \mp i W_{\mu}^{2}\right), \\
\left(\begin{array}{c}
Z_{\mu} \\
A_{\mu}
\end{array}\right) & =\left(\begin{array}{rr}
\cos \theta_{W} & -\sin \theta_{W} \\
\sin \theta_{W} & \cos \theta_{W}
\end{array}\right)\left(\begin{array}{c}
W_{\mu}^{3} \\
B_{\mu}
\end{array}\right) \equiv\left(\begin{array}{rr}
c_{W} & -s_{W} \\
s_{W} & c_{W}
\end{array}\right)\left(\begin{array}{l}
W_{\mu}^{3} \\
B_{\mu}
\end{array}\right) .
\end{aligned}
$$

In this equation, we have introduced the weak mixing angle at tree-level whose sine and cosine, noted as $s_{W}$ and $c_{W}$, are defined with respect to the hypercharge $\left(g^{\prime}\right)$, weak $(g)$ and electromagnetic $(e)$ coupling constants,

$$
g^{\prime} c_{W}=g s_{W}=e
$$

First, it can be observed that some of the new physics operators induce a modification of the kinetic terms of the gauge and Higgs bosons, once the neutral component of the Higgs doublet gets its vacuum expectation value $v$. For instance, the $\mathcal{O}_{G}$ operator of eq. (2.5) leads to a variation of the gluon field strength tensor squared term given by

$$
\mathcal{O}_{G}=\frac{g_{s}^{2} \bar{c}_{g}}{m_{W}^{2}} \Phi^{\dagger} \Phi G_{\mu \nu}^{a} G_{a}^{\mu \nu} \quad \Rightarrow \quad-\frac{g_{s}^{2} \bar{c}_{g} v^{2}}{2 m_{W}^{2}} G_{\mu \nu}^{a} G_{a}^{\mu \nu}
$$

and the $\mathcal{O}_{H}$ operator implies an additional contribution to the Higgs boson $h$ kinetic term,

$$
\mathcal{O}_{H}=\frac{\bar{c}_{H}}{2 v^{2}} \partial^{\mu}\left[\Phi^{\dagger} \Phi\right] \partial_{\mu}\left[\Phi^{\dagger} \Phi\right] \quad \Rightarrow \quad \frac{\bar{c}_{H}}{2} \partial^{\mu} h \partial_{\mu} h
$$

\footnotetext{
${ }^{1}$ The interfaces take care of discarding each vertex that is not compliant with the relevant program.
} 
Appropriate field redefinitions (at the first order in the effective couplings) are then required in order to bring back the various kinetic terms to their canonical forms, and read

$$
\begin{aligned}
& h \rightarrow h\left[1-\frac{1}{2} c_{H}\right], \\
& g_{\mu}^{a} \rightarrow g_{\mu}^{a}\left[1+\frac{\bar{c}_{g} g_{s}^{2} v^{2}}{m_{W}^{2}}\right], \\
& Z_{\mu} \rightarrow Z_{\mu}\left[1+\frac{\bar{c}_{\gamma} g^{2} s_{W}^{4} v^{2}}{c_{W}^{2} m_{W}^{2}}\right], \\
& A_{\mu} \rightarrow A_{\mu}\left[1+\frac{\bar{c}_{\gamma} e^{2} v^{2}}{m_{W}^{2}}\right]-Z_{\mu}\left[\frac{2 \bar{c}_{\gamma} s_{W} e^{2} v^{2}}{c_{W} m_{W}^{2}}\right] .
\end{aligned}
$$

While in our operator basis convention, the mass of the $W$-boson is agnostic of any new physics effect, there is an impact on both the $Z$-boson and Higgs-boson masses,

$$
\begin{aligned}
& m_{Z}^{2}=\frac{g^{2} v^{2}}{4 c_{w}^{2}}\left[1-\bar{c}_{T}+\frac{8 \bar{c}_{\gamma} s_{W}^{4}}{c_{W}^{2}}\right], \\
& m_{H}^{2}=2 v^{2} \lambda\left[1+\frac{13}{8} \bar{c}_{6}-\bar{c}_{H}\right] .
\end{aligned}
$$

In this last relation, we have made use of the minimization condition of the Higgs potential that reads, again in the first order in the effective couplings,

$$
v^{2}=\frac{\mu^{2}}{\lambda}\left[1-\frac{1}{2} \bar{c}_{6}\right] .
$$

These considerations and simplicity have motivated us to choose the electroweak input parameters that are used in the FeynRules model file. They consist in the mass of the $W$-boson $m_{W}$, the Fermi constant $G_{F}$, the electromagnetic coupling constant $\alpha$, as well as the Higgs-boson mass $m_{H}$. The field redefinitions of eq. (2.21), the replacement of the internal parameters in terms of the external ones and the removal of any term of higher order that is possibly arising from the procedure sketched above have been fully automated.

One could have implemented the effective Lagrangian of section 2.1 directly in the mass basis. This choice, adopted for instance in ref. [46], is in general clearer when one aims to properly estimate new physics effects that can be hinted for by a given physical observable. Several of the effective operators introduced in the previous section can indeed give rise to the same interaction in the mass basis, with the same Lorentz structure, so that it is not straightforward, if not impossible, to map a single measurement to a single operator. Moreover, a Lagrangian expressed in the mass basis also offers an easy way to be generalized by including appropriate form factors to model new physics effects. We consequently dedicate this section to a proper comparison of the two approaches, linking the effective Lagrangian of section 2.1 that is expressed entirely in the gauge basis to its counterpart as read off in terms of mass-eigenstates after electroweak symmetry breaking.

We now turn to the investigation of the interaction terms of the Lagrangian. Focusing on the terms related to the Higgs sector one writes

$$
\mathcal{L}_{\text {Higgs }}=\mathcal{L}^{(3)}+\mathcal{L}^{(4)}+\mathcal{L}^{(5)}+\mathcal{L}^{(6)},
$$




\begin{tabular}{|l|l|l|}
\hline Eq. (2.25) & Ref. [46] & Section 2.1 \\
\hline$g_{h g g}$ & $c_{\alpha} \kappa_{H g g} g_{H g g}$ & $g_{H}-\frac{4 \bar{c}_{g} g_{s}^{2} v}{m_{W}^{2}}$ \\
$\tilde{g}_{h g g}$ & $s_{\alpha} \kappa_{A g g} g_{A g g}$ & $-\frac{4 \tilde{c}_{g} g_{s}^{2} v}{m_{W}^{2}}$ \\
$g_{h \gamma \gamma}$ & $c_{\alpha} \kappa_{H \gamma \gamma} g_{H \gamma \gamma}$ & $a_{H}-\frac{8 g \bar{c}_{\gamma} s_{W}^{2}}{m_{W}}$ \\
$\tilde{g}_{h \gamma \gamma}$ & $s_{\alpha} \kappa_{A \gamma \gamma} g_{A \gamma \gamma}$ & $-\frac{8 g \tilde{c}_{\gamma} s_{W}^{2}}{m_{W}}$ \\
$g_{h z z}^{(1)}$ & $\frac{1}{\Lambda} c_{\alpha} \kappa_{H Z Z}$ & $\frac{2 g}{c_{W}^{2} m_{W}}\left[\bar{c}_{H B} s_{W}^{2}-4 \bar{c}_{\gamma} s_{W}^{4}+c_{W}^{2} \bar{c}_{H W}\right]$ \\
$\tilde{g}_{h z z}$ & $\frac{1}{\Lambda} s_{\alpha} \kappa_{A Z Z}$ & $\frac{2 g}{c_{W}^{2} m_{W}}\left[\tilde{c}_{H B} s_{W}^{2}-4 \tilde{c}_{\gamma} s_{W}^{4}+c_{W}^{2} \tilde{c}_{H W}\right]$ \\
$g_{h z z}^{(2)}$ & $\frac{1}{\Lambda} c_{\alpha} \kappa_{H \partial Z}$ & $\frac{g}{c_{W}^{2} m_{W}}\left[\left(\bar{c}_{H W}+\bar{c}_{W}\right) c_{W}^{2}+\left(\bar{c}_{B}+\bar{c}_{H B}\right) s_{W}^{2}\right]$ \\
$g_{h z z}^{(3)}$ & $c_{\alpha} \kappa_{\mathrm{SM}} g_{H Z Z}$ & $\frac{g m_{W}}{c_{W}^{2}}\left[1-\frac{1}{2} \bar{c}_{H}-2 \bar{c}_{T}+8 \bar{c}_{\gamma} \frac{s_{W}^{4}}{\left.c_{W}^{W}\right]}\right.$ \\
$g_{h a z}^{(1)}$ & $c_{\alpha} \kappa_{H Z \gamma} g_{H Z \gamma}$ & $\frac{g s_{W}}{c_{W} m_{W}}\left[\bar{c}_{H W}-\bar{c}_{H B}+8 \bar{c}_{\gamma} s_{W}^{2}\right]$ \\
$\tilde{g}_{h a z}$ & $s_{\alpha} \kappa_{A Z \gamma} g_{A Z \gamma}$ & $\frac{g s_{W}}{c_{W} m_{W}}\left[\tilde{c}_{H W}-\tilde{c}_{H B}+8 \tilde{c}_{\gamma} s_{W}^{2}\right]$ \\
$g_{h a z}^{(2)}$ & $\frac{1}{\Lambda} c_{\alpha} \kappa_{H \partial \gamma}$ & $\frac{g s_{W}}{c_{W} m_{W}}\left[\bar{c}_{H W}-\bar{c}_{H B}-\bar{c}_{B}+\bar{c}_{W}\right]$ \\
$g_{h w w}^{(1)}$ & $\frac{1}{\Lambda} c_{\alpha} \kappa_{H W W}$ & $\frac{2 g}{m_{W}} \bar{c}_{H W}$ \\
$\tilde{g}_{h w w}$ & $\frac{1}{\Lambda} s_{\alpha} \kappa_{A W W}$ & $\frac{2 g}{m_{W}} \tilde{c}_{H W}$ \\
$g_{h w w}^{(2)}$ & $\frac{1}{\Lambda} c_{\alpha} \kappa_{H \partial W}$ & $\frac{g}{m_{W}}\left[\bar{c}_{W}+\bar{c}_{H W}\right]$ \\
\hline
\end{tabular}

Table 1. Coupling strengths of the interactions of a Higgs boson with a vector boson pair. We present the relations between the Lagrangian parameters introduced in eq. (2.25) (first column), where the Lagrangian is expressed in the mass basis, and those associated with the operators of section 2.1 expressed in the gauge basis (third column). The Standard Model contributions to the Higgs boson to two photons (gluons) vertex $a_{H}\left(g_{H}\right)$ have been explicitly indicated. We relate these parameters to those employed in the Lagrangian description of ref. [46] in the second column of the table.

where $\mathcal{L}_{i}$ denotes the set of $i$-point interactions involving at least one Higgs boson. For the sake of the example, we only work out explicitly the structure of the interactions which are the more relevant for the phenomenology of the Higgs sector at the LHC, namely the three-point and four-point interactions involving at least one Higgs field. Vertices involving a higher number of external legs are in general related to processes associated with smaller cross sections, making them difficult to probe. We therefore refer to the FEYNRules implementation for their explicit form in the mass basis.

In the unitarity gauge and in the mass basis, the $\mathcal{L}_{3}$ Lagrangian reads

$$
\begin{aligned}
\mathcal{L}_{3}= & -\frac{m_{H}^{2}}{2 v} g_{h h h}^{(1)} h^{3}+\frac{1}{2} g_{h h h}^{(2)} h \partial_{\mu} h \partial^{\mu} h \\
& -\frac{1}{4} g_{h g g} G_{\mu \nu}^{a} G_{a}^{\mu \nu} h-\frac{1}{4} \tilde{g}_{h g g} G_{\mu \nu}^{a} \tilde{G}^{\mu \nu} h-\frac{1}{4} g_{h \gamma \gamma} F_{\mu \nu} F^{\mu \nu} h-\frac{1}{4} \tilde{g}_{h \gamma \gamma} F_{\mu \nu} \tilde{F}^{\mu \nu} h \\
& -\frac{1}{4} g_{h z z}^{(1)} Z_{\mu \nu} Z^{\mu \nu} h-g_{h z z}^{(2)} Z_{\nu} \partial_{\mu} Z^{\mu \nu} h+\frac{1}{2} g_{h z z}^{(3)} Z_{\mu} Z^{\mu} h-\frac{1}{4} \tilde{g}_{h z z} Z_{\mu \nu} \tilde{Z}^{\mu \nu} h
\end{aligned}
$$




\begin{tabular}{|l|l|}
\hline Eq. (2.25) - Eq. (2.26) & Section 2.1 \\
\hline$g_{h h h}^{(1)}$ & $1+\frac{7}{8} \bar{c}_{6}-\frac{1}{2} \bar{c}_{H}$ \\
$g_{h h h}^{(2)}$ & $\frac{g}{m_{W}} \bar{c}_{H}$ \\
$g_{h h h h}^{(1)}$ & $1+\frac{47}{8} \bar{c}_{6}-\bar{c}_{H}$ \\
$g_{h h h h}^{(2)}$ & $\frac{g^{2}}{4 m_{W}^{2}} \bar{c}_{H}$ \\
\hline
\end{tabular}

Table 2. Multiple Higgs interactions. We present the relations between the Lagrangian parameters introduced in eq. (2.25) and eq. (2.26), where the Lagrangian is expressed in the mass basis, and those associated with the operators of section 2.1 expressed in the gauge basis.

$$
\begin{aligned}
& -\frac{1}{2} g_{h a z}^{(1)} Z_{\mu \nu} F^{\mu \nu} h-\frac{1}{2} \tilde{g}_{h a z} Z_{\mu \nu} \tilde{F}^{\mu \nu} h-g_{h a z}^{(2)} Z_{\nu} \partial_{\mu} F^{\mu \nu} h-\frac{1}{2} g_{h w w}^{(1)} W^{\mu \nu} W_{\mu \nu}^{\dagger} h \\
& -\left[g_{h w w}^{(2)} W^{\nu} \partial^{\mu} W_{\mu \nu}^{\dagger} h+\text { h.c. }\right]+g\left(1-\frac{1}{2} \bar{c}_{H}\right) m_{W} W_{\mu}^{\dagger} W^{\mu} h-\frac{1}{2} \tilde{g}_{h w w} W^{\mu \nu} \tilde{W}_{\mu \nu}^{\dagger} h \\
& -\left[\tilde{y}_{u} \frac{1}{\sqrt{2}}\left[\bar{u} P_{R} u\right] h+\tilde{y}_{d} \frac{1}{\sqrt{2}}\left[\bar{d} P_{R} d\right] h+\tilde{y}_{\ell} \frac{1}{\sqrt{2}}\left[\bar{\ell} P_{R} \ell\right] h+\text { h.c. }\right]
\end{aligned}
$$

where the flavor indices of the fermions are understood and where we have introduced the $W$-boson, $Z$-boson and photon field strength tensors, $W_{\mu \nu}, Z_{\mu \nu}$ and $F_{\mu \nu}$. As already mentioned above and in ref. [46], this form of Lagrangian is sufficient to characterize all Higgs properties in a non-ambiguous way. This contrasts with the initial set of operators in the gauge basis which leads to additional structures that can be removed after several integrations by parts. In addition to all the operators already included in the Lagrangian of ref. [46], i.e., the Higgs to diboson couplings defined in table 1 and the Higgs to fermions interactions of table 3, we also include the triple Higgs interactions presented in table 2 . Furthermore, the tables also contain a translation dictionary linking our free parameters to the general Lagrangian employed in ref. [46].

The set of four-point interactions involving one or several Higgs fields is deduced from

$$
\begin{aligned}
\mathcal{L}_{4}= & -\frac{m_{H}^{2}}{8 v^{2}} g_{h h h h}^{(1)} h^{4}+\frac{1}{2} g_{h h h h}^{(2)} h^{2} \partial_{\mu} h \partial^{\mu} h-\frac{1}{8} g_{h h g g} G_{\mu \nu}^{a} G_{a}^{\mu \nu} h^{2}-\frac{1}{8} \tilde{g}_{h h g g} G_{\mu \nu}^{a} \tilde{G}_{a}^{\mu \nu} h^{2} \\
& -\frac{1}{8} g_{h h \gamma \gamma} F_{\mu \nu} F^{\mu \nu} h^{2}-\frac{1}{8} \tilde{g}_{h h \gamma \gamma} F_{\mu \nu} \tilde{F}^{\mu \nu} h^{2}-\frac{1}{8} g_{h h z z}^{(1)} Z_{\mu \nu} Z^{\mu \nu} h^{2}-\frac{1}{8} \tilde{g}_{h h z z} Z_{\mu \nu} \tilde{Z}^{\mu \nu} h^{2} \\
& -\frac{1}{2} g_{h h z z}^{(2)} Z_{\nu} \partial_{\mu} Z^{\mu \nu} h^{2}+\frac{1}{4} g_{h h z z}^{(3)} Z_{\mu} Z^{\mu} h^{2}-\frac{1}{4} g_{h h a z}^{(1)} Z_{\mu \nu} F^{\mu \nu} h^{2}-\frac{1}{4} \tilde{g}_{h h a z} Z_{\mu \nu} \tilde{F}^{\mu \nu} h^{2} \\
& -\frac{1}{2} g_{h h a z}^{(2)} Z_{\nu} \partial_{\mu} F^{\mu \nu} h^{2}-\frac{1}{4} g_{h h w w}^{(1)} W^{\mu \nu} W_{\mu \nu}^{\dagger} h^{2}-\frac{1}{4} \tilde{g}_{h h w w} W^{\mu \nu} \tilde{W}_{\mu \nu}^{\dagger} h^{2} \\
& -\frac{1}{2}\left[g_{h h w w}^{(2)} W^{\nu} \partial^{\mu} W_{\mu \nu}^{\dagger} h^{2}+\text { h.c. }\right]+\frac{1}{4} g^{2}\left(1-\bar{c}_{H}\right) W_{\mu}^{\dagger} W^{\mu} h^{2}-i g_{h a w w}^{(1)} F^{\mu \nu} W_{\mu} W_{\nu}^{\dagger} h \\
& +\left[i g_{h a w w}^{(2)} W^{\mu \nu} A_{\mu} W_{\nu}^{\dagger} h+\text { h.c. }\right]+i g_{h a w w}^{(3)} A_{\mu} W_{\nu} W_{\rho}^{\dagger}\left[\eta^{\mu \rho} \partial^{\nu} h-\eta^{\mu \nu} \partial^{\rho} h\right] \\
& +i \tilde{g}_{h a w w}^{(1)} \tilde{F}^{\mu \nu} W_{\mu} W_{\nu}^{\dagger} h+\left[i \tilde{g}_{h a w w}^{(2)} \tilde{W}^{\mu \nu} A_{\mu} W_{\nu}^{\dagger} h+\text { h.c. }\right] \\
& -i g_{h z w w}^{(1)} Z^{\mu \nu} W_{\mu} W_{\nu}^{\dagger} h+\left[i g_{h z w w}^{(2)} W^{\mu \nu} Z_{\mu} W_{\nu}^{\dagger} h+\text { h.c. }\right] \\
& +i \tilde{g}_{h z w w}^{(1)} \tilde{Z}^{\mu \nu} W_{\mu} W_{\nu}^{\dagger} h-\left[i \tilde{g}_{h z w w}^{(2)} \tilde{W}^{\mu \nu} Z_{\mu} W_{\nu}^{\dagger} h+\text { h.c. }\right]
\end{aligned}
$$




\begin{tabular}{|c|c|c|}
\hline Eq. (2.25) - Eq. (2.26) & Ref. [46] & Section 2.1 \\
\hline$\tilde{y}_{u}$ & $c_{\alpha} \kappa_{\text {Нии }} g_{\text {Нии }}$ & $y_{u}\left[1-\frac{1}{2} \bar{c}_{H}+\frac{3}{2} \bar{c}_{u}\right]$ \\
\hline$\tilde{y}_{d}$ & $c_{\alpha} \kappa_{H d d} g_{H d d}$ & $y_{d}\left|1-\frac{1}{2} \bar{c}_{H}+\frac{3}{2} \bar{c}_{d}\right|$ \\
\hline$\tilde{y}_{\ell}$ & $c_{\alpha} \kappa_{H \ell \ell} g_{H \ell \ell}$ & $y_{\ell}\left[1-\frac{1}{2} \bar{c}_{H}+\frac{3}{2} \bar{c}_{\ell}\right]$ \\
\hline $\bar{y}_{u}$ & - & $\frac{y_{u}}{v} \frac{3}{2} \bar{c}_{u}$ \\
\hline $\bar{y}_{d}$ & - & $\frac{y_{d}}{v} \frac{3}{2} \bar{c}_{d}$ \\
\hline $\bar{y}_{\ell}$ & - & $\frac{y \ell}{v} \frac{3}{2} \bar{c}_{\ell}$ \\
\hline$\left\{g_{h z u u}^{(L)}, g_{h z u u}^{(R)}\right\}$ & - & $\frac{g}{c_{W} v}\left\{\bar{c}_{H Q}-\bar{c}_{H Q}^{\prime}, \bar{c}_{H u}\right\}$ \\
\hline$\left\{g_{h z d d}^{(L)}, g_{h z d d}^{(R)}\right\}$ & - & $\frac{g}{c_{W} v}\left\{\bar{c}_{H Q}+\bar{c}_{H Q}^{\prime}, \bar{c}_{H d}\right\}$ \\
\hline$\left\{g_{h z \ell \ell}^{(L)}, g_{h z \ell \ell}^{(R)}\right\}$ & - & $\frac{g}{c_{W} v}\left\{\bar{c}_{H L}+\bar{c}_{H L}^{\prime}, \frac{1}{2} \bar{c}_{e}\right\}$ \\
\hline$g_{h z \nu \nu}$ & - & $\frac{g}{c_{W} v}\left[\bar{c}_{H L}-\bar{c}_{H L}^{\prime}\right]$ \\
\hline$\left\{g_{h w u d}^{(L)}, g_{h w u d}^{(R)}\right\}$ & - & $\frac{\sqrt{2} g}{v}\left\{\bar{c}_{H Q}^{\prime} V^{\mathrm{CKM}}, \bar{c}_{H u d}\right\}$ \\
\hline$g_{h w \nu \ell}$ & - & $\frac{\sqrt{2} g}{v} \bar{c}_{H L}^{\prime}$ \\
\hline$g_{h \gamma u u}^{(\partial)}$ & - & $\frac{\sqrt{2} g s_{W}}{m_{W}^{2}} y_{u}\left[\bar{c}_{u B}+\bar{c}_{u W}\right]$ \\
\hline$g_{h \gamma d d}^{(\partial)}$ & - & $\frac{\sqrt{2} g s_{W}}{m_{W}^{2}} y_{d}\left[\bar{c}_{d B}-\bar{c}_{d W}\right]$ \\
\hline$g_{h \gamma \ell \ell}^{(\partial)}$ & - & $\frac{\sqrt{2} g s_{W}}{m_{W}^{2}} y_{\ell}\left[\bar{c}_{e B}-\bar{c}_{e W}\right]$ \\
\hline$g_{h z u u}^{(\partial)}$ & - & $\frac{\sqrt{2} g}{c_{W} m_{W}^{2}} y_{u}\left[\bar{c}_{u W} c_{W}^{2}-\bar{c}_{u B} s_{W}^{2}\right]$ \\
\hline$g_{h z d d}^{(\partial)}$ & - & $\frac{\sqrt{2} g}{c_{W} m_{W}^{2}} y_{d}\left[-\bar{c}_{d W} c_{W}^{2}-\bar{c}_{d B} s_{W}^{2}\right]$ \\
\hline$g_{h z \ell \ell}^{(\partial)}$ & - & $\frac{\sqrt{2} g}{c_{W} m_{W}^{2}} y_{\ell}\left[-\bar{c}_{e W} c_{W}^{2}-\bar{c}_{e B} s_{W}^{2}\right]$ \\
\hline$\left\{g_{h w u d}^{(\partial L)}, g_{h w u d}^{(\partial R)}\right\}$ & - & $\frac{2 g}{m_{W}^{2}}\left\{y_{u}^{\dagger} V^{\mathrm{CKM}} \bar{c}_{u W}, V^{\mathrm{CKM}} y_{d} \bar{c}_{d W}\right\}$ \\
\hline$g_{h w \nu \ell}^{(\partial)}$ & - & $\frac{2 g}{m_{W}^{2}} y_{\ell} \bar{c}_{e W}$ \\
\hline$g_{\text {hguu }}^{(\partial)}$ & - & $\frac{2 \sqrt{2} g_{s}}{m_{W}^{2}} y_{u} \bar{c}_{u G}$ \\
\hline$g_{h g d d}^{(\partial)}$ & - & $\frac{2 \sqrt{2} g_{s}}{m_{W}^{2}} y_{d} \bar{c}_{d G}$ \\
\hline
\end{tabular}

Table 3. Coupling strengths of the interactions of one or several Higgs boson(s) with a fermion pair and possibly an additional gauge boson. We present the relations between the Lagrangian parameters introduced in eq. (2.25) (first column), where the Lagrangian is expressed in the mass basis, and those associated with the operators of section 2.1 expressed in the gauge basis (third column). In our notations, $V^{\text {СKM }}$ denotes the CKM mixing matrix. Concerning the three-point interactions, we relate our parameters to those of the Lagrangian description of ref. [46] in the second column of the table. 


$$
\begin{aligned}
& -i g_{h z w w}^{(3)} Z_{\mu} W_{\nu} W_{\rho}^{\dagger}\left[\eta^{\mu \rho} \partial^{\nu} h-\eta^{\mu \nu} \partial^{\rho} h\right] \\
& -\left[\bar{y}_{u} \frac{1}{\sqrt{2}}\left[\bar{u} P_{R} u\right] h^{2}+\bar{y}_{d} \frac{1}{\sqrt{2}}\left[\bar{d} P_{R} d\right] h^{2}+\bar{y}_{\ell} \frac{1}{\sqrt{2}}\left[\bar{\ell} P_{R} \ell\right] h^{2}+\text { h.c. }\right] \\
& -\bar{u} \gamma^{\mu}\left[g_{h z u u}^{(L)} P_{L}+g_{h z u u}^{(R)} P_{R}\right] u Z_{\mu} h-\bar{d} \gamma^{\mu}\left[g_{h z d d}^{(L)} P_{L}+g_{h z d d}^{(R)} P_{R}\right] d Z_{\mu} h \\
& -\bar{\ell} \gamma^{\mu}\left[g_{h z \ell \ell}^{(L)} P_{L}+g_{h z \ell \ell}^{(R)} P_{R}\right] \ell Z_{\mu} h-\bar{\nu} \gamma^{\mu}\left[g_{h z \nu \nu} P_{L}\right] \nu Z_{\mu} h \\
& -\left[\bar{u} \gamma^{\mu}\left[g_{h w u d}^{(L)} P_{L}+g_{h w u d}^{(R)} P_{R}\right] d W_{\mu} h+\bar{\nu} \gamma^{\mu}\left[g_{h w \nu \ell} P_{L}\right] \ell W_{\mu} h+\text { h.c. }\right] \\
& -\left[g_{h \gamma u u}^{(\partial)}\left[\bar{u} \gamma^{\mu \nu} P_{R} u\right]+g_{h \gamma d d}^{(\partial)}\left[\bar{d} \gamma^{\mu \nu} P_{R} d\right]+g_{h \gamma \ell \ell}^{(\partial)}\left[\bar{\ell} \gamma^{\mu \nu} P_{R} \ell\right]+\text { h.c. }\right] F_{\mu \nu} h \\
& -\left[g_{h z u u}^{(\partial)}\left[\bar{u} \gamma^{\mu \nu} P_{R} u\right]+g_{h z d d}^{(\partial)}\left[\bar{d} \gamma^{\mu \nu} P_{R} d\right]+g_{h z \ell \ell}^{(\partial)}\left[\bar{\ell} \gamma^{\mu \nu} P_{R} \ell\right]+\text { h.c. }\right] Z_{\mu \nu} h \\
& -\left[\bar{u} \gamma^{\mu \nu}\left[g_{h w u d}^{(\partial L)} P_{L}+g_{h w u d}^{(\partial R)} P_{R}\right] d W_{\mu \nu}+g_{h w \nu \ell}^{(\partial)} \bar{\nu} \gamma^{\mu \nu} P_{R} \ell W_{\mu \nu}+\text { h.c. }\right] h \\
& -\left[g_{h g u u}^{(\partial)}\left[\bar{u} T_{a} \gamma^{\mu \nu} P_{R} u\right]+g_{h g d d}^{(\partial)}\left[\bar{d} T_{a} \gamma^{\mu \nu} P_{R} d\right]+\text { h.c. }\right] G_{\mu \nu}^{a} h,
\end{aligned}
$$

where the free parameters are given in the various tables of this section. Hence, table 3 contains the parameters related to the interactions among one Higgs boson $h$, a fermionantifermion pair and possibly an additional gauge boson, while table 2 and table 4 are respectively dedicated to the Higgs boson self-interactions and to its interactions with vector bosons. We recall that some of the interaction vertices among a single Higgs field and three gluons were already included in the Lagrangian of eq. (2.25) through the gluon field strength tensor and its dual. Consequently, these are omitted from eq. (2.26). Along the same line, we include in a gauge-invariant way higher-dimensional Higgs boson and gluon vertices.

Both Lagrangians $\mathcal{L}_{3}$ and $\mathcal{L}_{4}$ not only exhibit Lorentz structures common with the Standard Model interactions, but also some novel ones. For instance, focusing on the Higgs to $W$-boson trilinear interactions, the complete Feynman rule reads

$$
\begin{array}{ll}
\underbrace{W_{u}^{+}\left(p_{2}\right)}_{W_{v}^{-}\left(p_{3}\right)} h\left(p_{1}\right) & i\left[\eta^{\mu \nu}\left(g m_{W}+g_{h w w}^{(1)} p_{2} \cdot p_{3}+g_{h w w}^{(2)}\left(p_{2}^{2}+p_{3}^{2}\right)\right)\right. \\
\left.-g_{h w w}^{(1)} p_{2}^{\nu} p_{3}^{\mu}-g_{h w w}^{(2)}\left(p_{2}^{\nu} p_{2}^{\mu}+p_{3}^{\nu} p_{3}^{\mu}\right)-\epsilon^{\mu \nu \rho \sigma} \tilde{g}_{h w w} p_{2 \rho} p_{3 \sigma}\right],
\end{array}
$$

where only the component proportional to the metric is present in the Standard Model.

Finally, new higher-dimensional operators involving the Higgs field can also have implications on vertices describing the self-interactions of the gauge bosons, in particular once the neutral component of the $\mathrm{SU}(2)_{\mathrm{L}}$ doublet $\Phi$ gets its vacuum expectation values. These new contributions supplement those accounted for by the Lagrangians $\mathcal{L}_{C P}$ and $\mathcal{L}_{G}$ of eq. (2.9) and eq. (2.14) that we omit from the rest of the discussion of this subsection for brevity. The complete and lengthy expressions can however be obtained from the FEYNRULES implementation of the model. The new physics effects that are induced by all the other operators of the Lagrangian of eq. (2.4) are then given, including the Standard Model 


\begin{tabular}{|l|l|}
\hline Eq. $(2.26)$ & Section 2.1 \\
\hline$g_{\text {hhgg }}$ & $-\frac{4 \bar{c}_{g} g_{s}^{2}}{m_{W}^{2}}$ \\
$\tilde{g}_{\text {hhgg }}$ & $-\frac{4 \tilde{c}_{g} g_{s}^{2}}{m_{W}^{2}}$ \\
$g_{h h \gamma \gamma}$ & $-\frac{4 \bar{c}_{\gamma} g^{2} s_{W}^{2}}{m_{W}^{2}}$ \\
$\left\{\tilde{g}_{h h \gamma \gamma}, \tilde{g}_{h h z z}, \tilde{g}_{h h a z}, \tilde{g}_{h h w w}\right\}$ & $\frac{g}{2 m_{W}}\left\{\tilde{g}_{h \gamma \gamma}, \tilde{g}_{h z z}, \tilde{g}_{h a z}, \tilde{g}_{h w w}\right\}$ \\
$\left\{g_{h h z z}^{(1)}, g_{h h z z}^{(2)}, g_{h h a z}^{(1)}, g_{h h a z}^{(2)}, g_{h h w w}^{(1)}, g_{h h w w}^{(2)}\right\}$ & $\frac{g}{2 m_{W}}\left\{g_{h z z}^{(1)}, g_{h z z}^{(2)}, g_{h a z}^{(1)}, g_{h a z}^{(2)}, g_{h w w}^{(1)}, g_{h w w}^{(2)}\right\}$ \\
$g_{h h z z}^{(3)}$ & $\frac{g^{2}}{2 c_{W}^{2}}\left[1-6 \bar{c}_{T}-\bar{c}_{H}+8 \bar{c}_{\gamma} \frac{s_{W}^{4}}{c_{W}^{2}}\right]$ \\
$g_{\text {haww }}^{(1)}$ & $\frac{g^{2} s_{W}}{m_{W}}\left[2 \bar{c}_{W}+\bar{c}_{H B}+\bar{c}_{H W}\right]$ \\
$\tilde{g}_{\text {haww }}^{(1)}$ & $\frac{g^{2} s_{W}}{m_{W}}\left[\tilde{c}_{H W}-\tilde{c}_{H B}\right]$ \\
$g_{h a w w}^{(2)}$ & $\frac{2 g^{2} s_{W}}{m_{W}} \bar{c}_{W}$ \\
$\tilde{g}_{\text {haww }}^{(2)}$ & $\frac{g^{2} s_{W}}{m_{W}} \tilde{c}_{H W}$ \\
$g_{\text {haww }}^{(3)}$ & $\frac{g^{2} s_{W}}{m_{W}}\left[\bar{c}_{H W}+\bar{c}_{W}\right]$ \\
$g_{h z w w}^{(1)}$ & $\frac{g^{2}}{c_{W} m_{W}}\left[\bar{c}_{H W} c_{W}^{2}-\bar{c}_{H B} s_{W}^{2}+\bar{c}_{W}\left(3-2 s_{W}^{2}\right)\right]$ \\
$\tilde{g}_{h z w w}^{(1)}$ & $\frac{g^{2}}{c_{W} m_{W}}\left[\tilde{c}_{H W}\left(2-s_{W}^{2}\right)+\tilde{c}_{H B} s_{W}^{2}\right]$ \\
$g_{h z w w}^{(2)}$ & $\frac{g^{2}}{c_{W} m_{W}}\left[\bar{c}_{H W}+\bar{c}_{W}\left(3-2 s_{W}^{2}\right)\right]$ \\
$\tilde{g}_{h z w w}^{(2)}$ & $\frac{2 g^{2}}{m_{W}} c_{W} \tilde{c}_{H W}$ \\
$g_{h z w w}^{(3)}$ & $\frac{g^{2}}{c_{W} m_{W}} s_{W}^{2}\left[\bar{c}_{H W}+\bar{c}_{W}\right]$ \\
\hline
\end{tabular}

Table 4. Quartic interactions of one or several Higgs field with gauge bosons. We present the relations between the Lagrangian parameters introduced in eq. (2.26), where the Lagrangian is expressed in the mass basis, and those associated with the operators of section 2.1 expressed in the gauge basis.

contributions, by

$$
\begin{aligned}
\mathcal{L}_{3 V}= & {\left[i g_{a w w}^{(1)} W_{\mu \nu}^{\dagger} A^{\mu} W^{\nu}+\text { h.c. }\right]+i g_{a w w}^{(2)} F_{\mu \nu} W^{\mu} W^{\nu \dagger} } \\
& +\left[i g_{z w w}^{(1)} W_{\mu \nu}^{\dagger} Z^{\mu} W^{\nu}+\text { h.c. }\right]+i g_{z w w}^{(2)} Z_{\mu \nu} W^{\mu} W^{\nu \dagger}
\end{aligned}
$$

and

$$
\begin{aligned}
\mathcal{L}_{4 V}= & g_{w w w w}\left[W_{\mu} W^{\mu} W_{\nu}^{\dagger} W^{\nu \dagger}-W_{\mu} W^{\nu} W_{\nu}^{\dagger} W^{\mu \dagger}\right] \\
& +g_{a a w w}\left[A^{\mu} A_{\mu} W_{\nu}^{\dagger} W^{\nu}-A_{\mu} A^{\nu} W_{\nu}^{\dagger} W^{\mu}\right] \\
& +g_{z z w w}\left[Z^{\mu} Z_{\mu} W_{\nu}^{\dagger} W^{\nu}-Z_{\mu} Z^{\nu} W_{\nu}^{\dagger} W^{\mu}\right] \\
& +g_{a z w w}\left[A^{\mu} Z_{\mu} W_{\nu}^{\dagger} W^{\nu}-A_{\mu} Z^{\nu} W_{\nu}^{\dagger} W^{\mu}\right]
\end{aligned}
$$




\begin{tabular}{|l|l|}
\hline eq. (2.27) & Section 2.1 \\
\hline$g_{a w w}^{(1)}$ & $e\left[1-2 \bar{c}_{W}\right]$ \\
$g_{a w w}^{(2)}$ & $e\left[1-2 \bar{c}_{W}-\bar{c}_{H B}-\bar{c}_{H W}\right]$ \\
$g_{z w w}^{(1)}$ & $\frac{g}{c_{W}}\left[c_{W}^{2}-\bar{c}_{H W}+\left(2 s_{W}^{2}-3\right) \bar{c}_{W}\right]$ \\
$g_{z w w}^{(2)}$ & $\frac{g}{c_{W}}\left[c_{W}^{2}\left(1-\bar{c}_{H W}\right)+s_{W}^{2} \bar{c}_{H B}+\left(2 s_{W}^{2}-3\right) \bar{c}_{W}\right]$ \\
\hline
\end{tabular}

Table 5. Trilinear gauge interactions. We present the relations between the Lagrangian parameters introduced in eq. (2.27), where the Lagrangian is expressed in the mass basis, and those associated with the operators of section 2.1 expressed in the gauge basis.

\begin{tabular}{|l|l|}
\hline eq. (2.28) & Section 2.1 \\
\hline$g_{w w w w}$ & $\frac{g^{2}}{2}\left[1-2 \bar{c}_{H W}-4 \bar{c}_{W}\right]$ \\
$g_{\text {aaww }}$ & $e^{2}\left[-1+2 \bar{c}_{W}\right]$ \\
$g_{z z w w}$ & $g^{2}\left[-c_{W}^{2}+2 \bar{c}_{H W}+2\left(2-s_{W}^{2}\right) \bar{c}_{W}\right]$ \\
$g_{a z w w}$ & $\frac{2 g e}{c_{W}}\left[-c_{W}^{2}+\bar{c}_{H W}+\left(3-2 s_{W}^{2}\right) \bar{c}_{W}\right]$ \\
\hline
\end{tabular}

Table 6. Quartic gauge interactions. We present the relations between the Lagrangian parameters introduced in eq. (2.28), where the Lagrangian is expressed in the mass basis, and those associated with the operators of section 2.1 expressed in the gauge basis.

after splitting the different terms into three-point and four-point contributions, respectively, and neglecting any other vertex containing more than four external legs. We collect the coefficients of the different operators entering those Lagrangians in table 5 and table 6 and give their expressions in terms of the Wilson coefficients of the Higgs effective Lagrangian presented in section 2.1 .

\section{Experimental constraints on dimension-six effective operators}

The magnitude of the Wilson coefficients associated with the dimension-six operators introduced in section 2, and thus an estimate of their impact on physical observables, can be naively computed by a simple power counting [93,97]. In this way, each power of $\Phi$ leads to a $g_{N P} / M$ suppression factor, $M$ being the typical mass scale of the new physics sector and $g_{N P}$ the coupling strength of the new states to the Higgs field, while each derivative implies an additional reduction of $1 / M$. Additionally, in the framework of a given theory, specific operators can be generated at the one-loop level so that additional suppression can be foreseen. Sticking to tree-level, Wilson coefficients such as $\bar{c}_{H}, \bar{c}_{T}, \bar{c}_{6}$ or $\bar{c}_{\psi}$ are expected to be of the order of

$$
\bar{c}_{H}, \bar{c}_{T}, \bar{c}_{6}, \bar{c}_{\psi} \sim \mathcal{O}\left(\frac{g_{N P}^{2} v^{2}}{M^{2}}\right)
$$


and can therefore be quite large for strongly coupled new physics, while in contrast, the coefficients of operators such as $\bar{c}_{W}$ and $\bar{c}_{B}$ scale as

$$
\bar{c}_{B}, \bar{c}_{W} \sim \mathcal{O}\left(\frac{m_{W}^{2}}{M^{2}}\right)
$$

and are thus expected to be relatively suppressed or enhanced according to the value of the ratio $g / g_{N P}$.

In addition, the value of the 39 Wilson coefficients introduced in section 2 is experimentally constrained from various sources. These limits mainly arise from collider data (LEP, Tevatron, LHC), electric dipole moment measurements, rare decay bounds and results of several experiments dedicated to the measurement of the anomalous magnetic moments of the muon and the electron. An exhaustive list of the current bounds on the various coefficients of the considered dimension-six operators can be found in ref. [97] so that we omit it from the present manuscript.

Some of these constraints however only involve specific combinations of Wilson coefficients instead of a single coefficient. An example lies in the recasting, in the language of the Higgs effective Lagrangian of section 2.1, of the constraints originating from the electroweak precision parameters derived in ref. [142],

$$
\bar{c}_{T}\left(m_{Z}\right) \in[-1.5,2.2] \times 10^{-3} \quad \text { and } \quad\left(\bar{c}_{W}\left(m_{Z}\right)+\bar{c}_{B}\left(m_{Z}\right)\right) \in[-1.4,1.9] \times 10^{-3} .
$$

This still leaves open the possibility of a cancellation between two large $\bar{c}_{W}$ and $\bar{c}_{B}$ coefficients at the $Z$-pole. The possibility of such cancellations also holds for other coefficients, such as $\bar{c}_{H W}$ or $\bar{c}_{H B}$, for which there exists no strong bound from the LEP experiments. A more involved example arises from the strong limits on the quantity $\bar{c}_{W W}$ defined by a combination of several of the $\bar{c}_{i}$ coefficients,

$$
\bar{c}_{W W}=\bar{c}_{W}-\bar{c}_{B}+\bar{c}_{H B}-\bar{c}_{H W}+\frac{1}{4} \bar{c}_{\gamma}
$$

Existing data being too limited to allow for disentangling the individual effects of the different operators, this implies that no constraints can be really inferred in our choice of basis for the dimension-six operators.

Those considerations motivate us to avoid a careful design of a benchmark scenario theoretically motivated and not experimentally excluded, a task that can only be performed from a global fit of all data and considering all dimension-six operators, including additional four-fermion interactions, their one-loop mixings as well as field equations of motion linking our basis of operators to an extended one with redundant operators. We instead pick up, in section 4, several phenomenological examples illustrating possible usages of the Higgs effective field theory implementation achieved in this work, so that it could be possibly used for such global fits in future works.

\section{Phenomenological examples}

\subsection{Interactions between the Higgs boson and the electroweak gauge bosons}

As first examples of a possible usage of our implementation, we focus on the restricted set of operators implying modifications of the $h V V$ couplings between one single Higgs 
boson and a gauge-boson pair. New physics effects possibly arising in such interactions have been largely investigated in pioneering and more recent works prior the discovery of a Higgs boson by the ATLAS and CMS experiments [5-18, 20, 22-24, 26, 87-89, 91, 92, 95, 96, 143-145]. Within the last two years, they have been reassessed in the light of newer LHC data including the Higgs results [28-30, 34, 37, 45, 100-105, 146]. We reproduce and extend, in the rest of this subsection, some of these results by employing the MadGraph 5 package [129], using our FeynRules [106, 113] implementation to generate the necessary UFO model files [134]. The events have eventually been analyzed by means of MadAnalysis 5 [140, 141].

\subsubsection{Probing the custodial symmetry}

Among all the Wilson coefficients included in the Lagrangian of eq. (2.4), the $\bar{c}_{W}$ parameter has the specificity to imply modifications of both the $h Z Z$ and $h W W$ interactions simultaneously. Its value can therefore be probed by independent measurements of the Higgs boson properties in its $W W$ and $Z Z$ decay modes, when considering, e.g., leptonic weak boson decays,

$$
h \rightarrow Z^{*} Z^{(*)} \rightarrow 4 \ell \quad \text { and } \quad h \rightarrow W^{*} W^{(*)} \rightarrow \ell \nu \ell \nu .
$$

Analyzing the full set of LHC data describing collisions at center-of-mass energies of $7 \mathrm{TeV}$ and $8 \mathrm{TeV}$, considering those two decay patterns and a Higgs-boson production by gluon fusion, the ATLAS collaboration has recently ${ }^{2}$ reported a measurement [148] of the ratio of the two corresponding branching fractions estimated relatively to the Standard Model expectations,

$$
\lambda_{W Z}=\frac{\kappa_{W}}{\kappa_{Z}}
$$

with

$$
\kappa_{W}=\frac{\Gamma\left(h \rightarrow W^{*} W^{(*)}\right)}{\Gamma\left(h \rightarrow W^{*} W^{(*)}\right)_{S M}} \quad \text { and } \quad \kappa_{Z}=\frac{\Gamma\left(h \rightarrow Z^{*} Z^{(*)}\right)}{\Gamma\left(h \rightarrow Z^{*} Z^{(*)}\right)_{S M}} .
$$

Assuming a Higgs boson mass of $m_{h}=125.5 \mathrm{GeV}$, the quantity $\lambda_{W Z}$ has been found to lie in a range defined by

$$
\lambda_{W Z}=0.82 \pm 0.15
$$

at the $95 \%$ confidence level and when keeping the strength of the Higgs boson to two photon coupling as a free parameter. This last assumption allows for beyond the Standard Model effects in the $h \gamma \gamma$ interactions, the latter being in particular expected from a non-zero $\bar{c}_{W}$ parameter too (see table 1). Employing our framework, we theoretically estimate the two $\kappa$ parameters and the $\lambda_{W Z}$ quantity as

$$
\begin{aligned}
\kappa_{W} & =1+2.23 \bar{c}_{W}+1.27 \bar{c}_{W}^{2}, \\
\kappa_{Z} & =1+1.97 \bar{c}_{W}+1.00 \bar{c}_{W}^{2}, \\
\lambda_{W Z} & =1+0.28 \bar{c}_{W}-0.27 \bar{c}_{W}^{2},
\end{aligned}
$$

\footnotetext{
${ }^{2} \mathrm{~A}$ similar study has been performed by the CMS collaboration, but the results do not account for all 2011-2012 LHC data [147].
} 


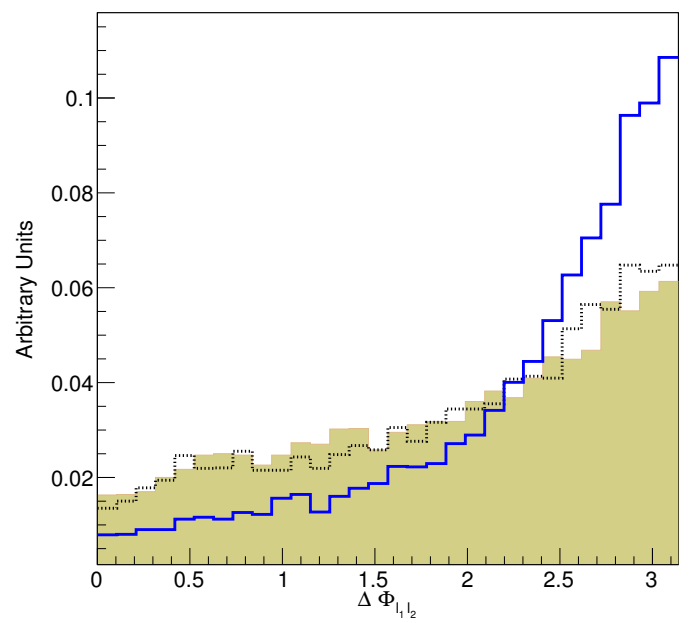

Figure 1. Distribution of the angular separation of the decay planes associated with each of the (possibly virtual) $Z$-bosons resulting from the decay of a Higgs boson produced in the gluon fusion mode. We show the Standard model distribution (green-solid histogram) to which we superimpose predictions associated with a non-zero $\bar{c}_{W}=0.3$ (black-dotted line) and -1.5 (blue-solid line) Wilson coefficient.

so that we extract, at the $95 \%$ confidence level,

$$
\bar{c}_{W} \in[-1.71,0.42],
$$

from the ATLAS results. This range is still largely compatible with the naive estimate computed as indicated in section 3 ,

$$
\bar{c}_{W} \simeq \mathcal{O}\left(\frac{v}{\Lambda}\right)^{2} \simeq 10^{-3}
$$

considering TeV scale new physics [97, 100-105] and also agrees with predictions performed by making use of the EHDECAY program [97], the extension of the HDECAY package [149, 150] in the framework of the Higgs effective field theory of section 2.1.

In the case both the $\bar{c}_{W}$ and $\bar{c}_{B}$ coefficients are non-vanishing, as suggested by the LEP limits constraining their difference, the additional effect of the $\bar{c}_{B}$ parameter on the $\kappa_{Z}$ quantity can be obtained by replacing $\bar{c}_{W}$ by $\bar{c}_{W}+\tan ^{2} \theta_{W} \bar{c}_{B}$ in eq. (4.5). The corresponding modification in the predictions for the $\lambda_{W Z}$ parameter is trivially obtained by, e.g., linearizing the new physics effects.

\subsubsection{Kinematics of the four-lepton system issued from a $h \rightarrow Z^{*} Z^{(*)}$ decay}

In addition to shifting the values of the Higgs signal strengths $\kappa_{W}$ and $\kappa_{Z}$, higher-dimensional operators also affect various kinematical distributions. In particular, several angular distributions related to the leptonic systems arising from decays of the Higgs boson into four fermions such as those introduced in eq. (4.1) can be drastically modified by new physics effects. Considering the $Z Z^{*}$ channel in the four-lepton mode where the entire final state 
can be reconstructed, we simulate LHC collisions at a center-of-mass energy of $14 \mathrm{TeV}$ yielding the production of a Higgs boson from a gluon-pair initial state, followed by its decay into four leptons,

$$
g g \rightarrow h \rightarrow Z^{*} Z^{(*)} \rightarrow\left(\ell_{1}^{-} \ell_{1}^{+}\right)\left(\ell_{2}^{-} \ell_{2}^{+}\right) .
$$

In our setup, each lepton is required to have a transverse momentum greater than $10 \mathrm{GeV}$, a pseudorapidity $|\eta|<2.5$ and to be isolated. We define lepton isolation by enforcing a minimum angular distance $\Delta R$ of 0.4 between any two of the produced particles. In our notations, $\Delta R=\sqrt{\Delta \varphi^{2}+\Delta \eta^{2}}$ with $\varphi$ denoting the azimuthal angle with respect to the beam direction.

The four-momenta of the four final state leptons can be seen as lying on three distinct planes, one of them being related to the decaying Higgs boson into two (possibly virtual) $Z$-bosons and the two other being associated with the decay products of each $Z$-boson. As it is customary in the literature $[5-18,20,22-24,28-30,34,45]$, we denote by $\theta_{1,2}$ the polar angles of the two leptons $\ell_{1,2}^{-}$in the rest frame of the respective decaying $Z$-bosons, and by $\Delta \phi_{\ell_{1}^{-} \ell_{2}^{-}}$the azimuthal angle between the planes formed by each lepton pair in the Higgs boson rest frame.

We show in figure 1 the angular distribution $\Delta \phi_{\ell_{1} \ell_{2}}$ for the pure Standard model case (green-solid histogram) to which we superimpose predictions obtained for two choices of non-vanishing $\bar{c}_{W}$ Wilson coefficient. These latter predictions also include Standard Model contributions with which new physics interferes. The black-dashed and blue-solid lines in the figure correspond to values of $\bar{c}_{W}=0.3$ and -1.5 , respectively. The Standard Model spectrum is expected to be a function of both $\cos \left(\Delta \phi_{\ell_{1} \ell_{2}}\right)$ and $\cos \left(2 \Delta \phi_{\ell_{1} \ell_{2}}\right)$ exhibiting a rather gentle slope. This known result $[17,151]$ is recovered by our predictions. In contrast, the structure of the $\mathcal{O}_{W}$ dimension-six operators modifies the coefficients of the polynomial in the cosines of the $\Delta \phi_{\ell_{1} \ell_{2}}$ angle, leading to a steeper dependence. The larger the value of $\bar{c}_{W}$, the steeper this function becomes. Consequently, this $\Delta \phi_{\ell_{1} \ell_{2}}$ distribution can clearly be used, if measured with a decent precision, to constrain the magnitude of the Wilson coefficient associated with the operator $\mathcal{O}_{W}$.

\subsubsection{Kinematics of the two-lepton system issued from a $h \rightarrow W^{*} W^{(*)}$ decay}

We now consider the $W W^{*}$ Higgs decay mode in the case both weak bosons decay leptonically,

$$
g g \rightarrow h \rightarrow W^{*} W^{(*)} \rightarrow 2 \ell 2 \nu,
$$

in the framework of LHC collisions at a center-of-mass energy of $14 \mathrm{TeV}$ and for similar lepton requirements as in the previous subsection. We study new physics effects arising from non-vanishing $\bar{c}_{W}$ and $\bar{c}_{H W}$ parameters in the spectrum of the angular distance $\Delta R$, in the $(\eta, \varphi)$ plane, among the two produced leptons. We have found that the resulting distribution is barely affected when the two Wilson coefficients of interest lie in the range $[-0.5,0.5]$. This feature is illustrated on the left panel of figure 2 in which we first compute the Standard Model expectation obtained when all the new physics parameters are set to zero (green-solid histogram) and then superimpose results obtained for the choices of 

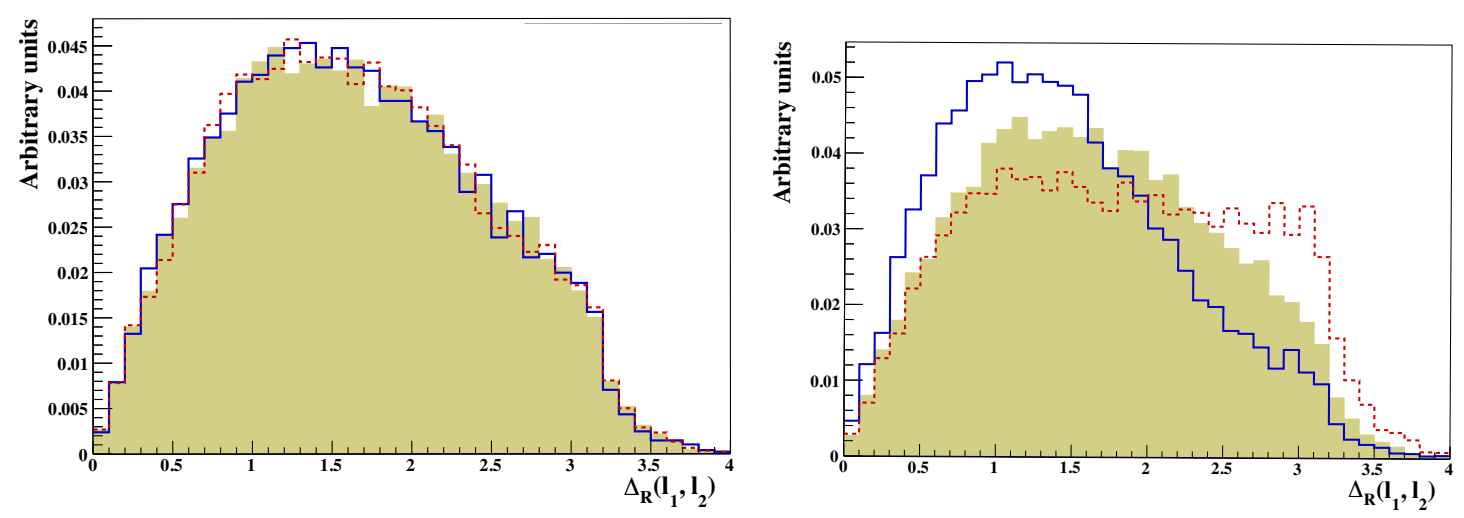

Figure 2. Distribution of the angular distance $\Delta R\left(\ell_{1}, \ell_{2}\right)$ between the two charged leptons originating from a pair of $W$-bosons issued from the decay of a Higgs boson produced via gluon fusion. The left panel of the figure illustrates that for $\bar{c}_{W}=0.05$ (red-dashed line) and $\bar{c}_{H W}=0.05$ (bluesolid line), no sizable effect beyond the SM predictions (green-solid histogram) can be expected. In contrast, the right panel of the figure shows that for larger values of the Wilson coefficients set to -1 , sensitive effects can be observed.

$\bar{c}_{H W}=0.5$ (blue-solid line) and $\bar{c}_{W}=0.05$ (red-dashed line). In contrast, for larger values of the Wilson coefficients, important differences in the distributions can be expected, as shown on the right panel of the figure for two representative scenarios with respectively $\bar{c}_{H W}=-1$ (blue-solid line) and $\bar{c}_{W}=-1$ (red-dashed line). It can be noted that according to the new physics benchmark scenarios the most striking effects can be either expected in the low $\Delta R$ region, or in the higher one.

\subsubsection{Correlations of new physics effects in the Higgs boson partial widths}

As already mentioned in section 2, one given dimension-six operator may affect several interactions of the Higgs boson to a pair of gauge bosons. This feature is employed in figure 3 where we compute different Higgs boson partial widths when considering a specific dimension-six operator, allowed to vary in the $[-1,1]$ range. The results of the different decay channels are then confronted to each other and we study the correlations among the corresponding partial widths. It can be seen that this information could be used in the future to constrain which Wilson coefficients are allowed to be non-negligible by data. It is however clear that this type of investigation consists only of a first step towards a global fit of a complete set of dimension-six operators. In addition, our results are compared in each of the treated cases to the Standard Model predictions represented by an orange dot.

In this way, we present on the upper-left panel of the figure the variations of the $h \rightarrow \gamma \gamma$ and $h \rightarrow Z \gamma$ partial widths for non-vanishing $C P$-conserving and $C P$-violating parameters $\bar{c}_{\gamma}$ and $\tilde{c}_{\gamma}$. We observe that those two channels do not offer a clear way to distinguish contributions arising from the two associated $\mathcal{O}_{\gamma}$ and $\tilde{\mathcal{O}}_{\gamma}$ operators. The situation is however drastically different when we focus, in the upper-right panel of the figure, on correlations possibly observable in the $h \rightarrow \gamma \gamma$ and $h \rightarrow Z Z$ channels and that are induced by variations of the same $\bar{c}_{\gamma}$ and $\tilde{c}_{\gamma}$ parameters. In this case, if one assumes precise measurements pointing towards physics beyond the Standard Model in one or the other 

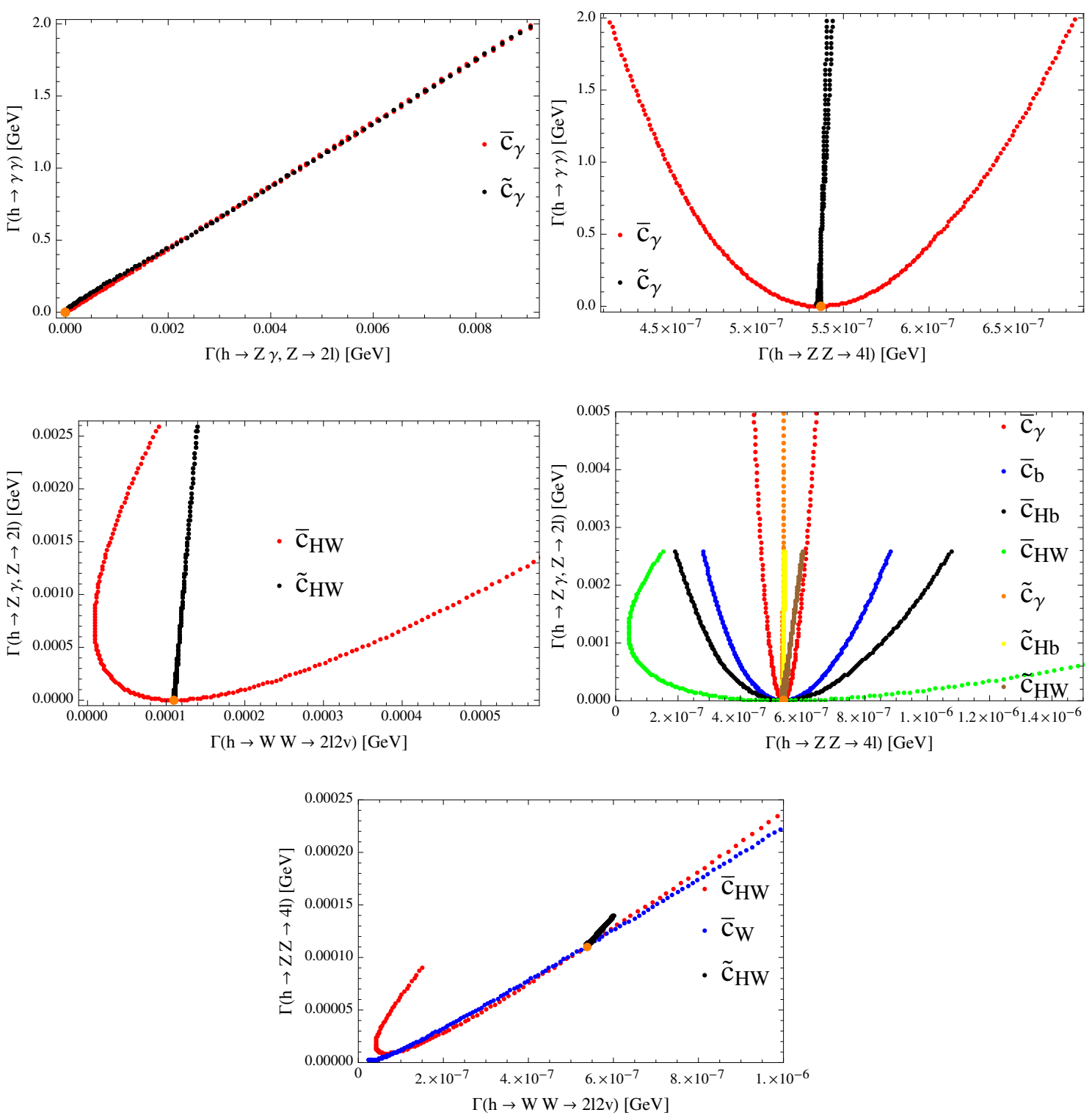

Figure 3. Correlations of several Higgs boson partial widths when different Wilson coefficients are chosen non-vanishing. In the upper-left (upper-right) panel of the figure, we investigate simultaneous new physics effects on the $h \rightarrow \gamma \gamma$ and $h \rightarrow Z \gamma(h \rightarrow Z Z)$ partial widths, in its central-left (central-right) panel on the $h \rightarrow Z \gamma$ and $h \rightarrow W W(h \rightarrow Z Z)$ partial widths and in its lower panel on the $h \rightarrow Z Z$ and $h \rightarrow W W$ partial widths. We indicate the Standard Model expectation by an orange dot.

Higgs decay modes (possibly in collisions at future LHC center-of-mass energies or even at future colliders), it can be observed that these measurements could help to get an handle on the structure of the operators responsible for the assumed observations. More involved cases are treated in the three other panels of the figure, where we confront predictions for several partial widths of the Higgs boson into different gauge-boson pairs for various choices of non-zero Wilson coefficients that are allowed to vary in the $[-1,1]$ range. For 


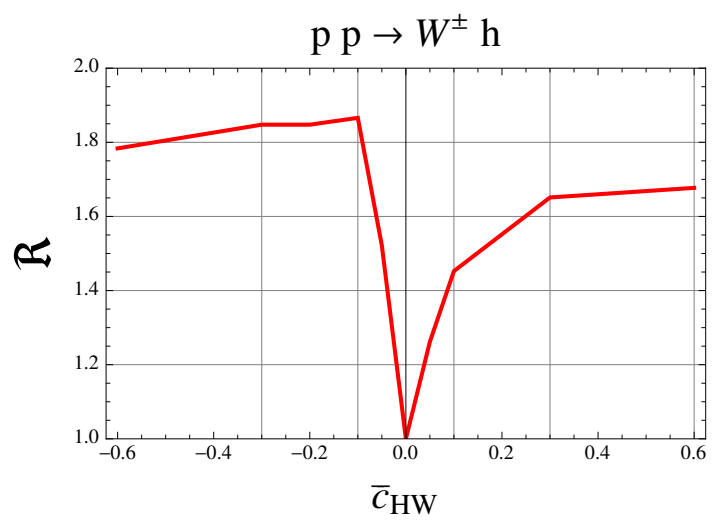

Figure 4. Double ratio $\mathcal{R}$ of total cross sections at $\sqrt{S}=8 \mathrm{TeV}$ and $14 \mathrm{TeV}$, as defined in eq. (4.11), given as a function of the value of the $\bar{c}_{H W}$ parameter for the process $p p \rightarrow W^{ \pm} h \rightarrow \ell \nu b \bar{b}$ at the LHC. No selection on the final state lepton and missing energy has been accounted for.

most of the cases, it is found that this method allows for distinguishing among the different operators that could model a specific new physics effect.

\subsection{Higgs boson production in association with a vector boson}

We now turn to the study of the production of a Higgs boson in association with a weak gauge boson

$$
p p \rightarrow V^{*} \rightarrow h V,
$$

with $V$ being either a $W$-boson or a $Z$-boson. The existing searches are classified according to the lepton multiplicity of the final state, i.e., in dileptonic, singly leptonic and zero-lepton channels. This offers the possibility to make use of the properties of the final state leptons possibly coming accompanied by missing transverse energy to reject the Standard Model background without affecting too much the Higgs signal as the latter could be searched for in its dominant decay mode to two jets originating from the fragmentation of $b$-quarks.

In this case, the final state kinematics differs from the one expected from gluon fusion which was discussed in the previous subsection [44, 152]. In gluon fusion, the Higgs is usually produced with a very little boost as the partonic center-of-mass energy is close to the Higgs-boson mass. Contrary, a two-body system comprised of a Higgs boson and a $W$ - or $Z$-boson is issued from an off-shell vector boson that can be seen as radiating a Higgs particle. This off-shellness allows for greater partonic center-of-mass energies, which subsequently increases the sensitivity to non-standard Lorentz structures in the interactions of the Higgs field as modeled by the effective operators of section 2.1. We exploit these features in the rest of this subsection.

\subsubsection{New physics effects in total cross sections for associated Higgs and gauge boson production}

Total rates in the associated production of a Higgs boson with a gauge boson [31, 153-155] are known to be a powerful handle to obtain information on beyond the Standard Model effects modeled by dimension-six operators. In particular, a class of variables consisting of 
double ratios of total cross sections has been recently identified as one of the key players for this task $[153,156]$. More into details, such quantities are defined as ratios of total cross sections at different center-of-mass energies, normalized to the corresponding Standard Model values. Focusing on present and future LHC collision center-of-mass energies $(\sqrt{S}=8 \mathrm{TeV}$ and $14 \mathrm{TeV})$, we investigate in this work the variable

$$
\mathcal{R} \equiv\left(\frac{\sigma(\sqrt{S}=14 \mathrm{TeV})}{\sigma(\sqrt{S}=8 \mathrm{TeV})}\right)_{\bar{c}_{i}} /\left(\frac{\sigma(\sqrt{S}=14 \mathrm{TeV})}{\sigma(\sqrt{S}=8 \mathrm{TeV})}\right)_{S M}
$$

where the subscript $\bar{c}_{i}$ indicates a computation of the cross section after including the effects of an higher-order operator associated with the Wilson coefficient $\bar{c}_{i}$. It is also believed that this type of variables could be useful in the case statistically relevant and separated information for collisions at center-of-mass energies of $13.5 \mathrm{TeV}$ and $14 \mathrm{TeV}$ would be available [157].

We illustrate the use of the variable introduced in eq. (4.11) in figure 4 by investigating the process

$$
p p \rightarrow W^{ \pm} h \rightarrow(\ell \nu)(b \bar{b}) .
$$

We show the dependence of $\mathcal{R}$ on the coefficient of $\bar{c}_{H W}$ which turns out to be quite steep when $\bar{c}_{H W}$ is of order $\mathcal{O}(0.1)$ or smaller, and smoother for larger (absolute) values of this Wilson coefficient. The results however largely depend on the selection requirements (on the final state lepton and missing transverse energy) of the corresponding analysis that could further accentuate the effect of the effective operator.

\subsubsection{Invariant mass of a two-body system constituted of a Higgs boson and a gauge boson}

The kinematical properties of the system formed by the massive vector boson $V$ and the Higgs boson $h$ may be modified by the presence in the Lagrangian of non-standard operators such as those introduced in section 2.1. In this context, one interesting observable consists of the invariant-mass distribution of the $V h$-system [31], as illustrated in figure 5 for proton-proton collisions at a center-of-mass energy of $14 \mathrm{TeV}$. We present in this figure invariant-mass $m_{V h}$ spectra computed at the parton-level, i.e., without accounting for gauge-boson and Higgs-boson decays, and compare the Standard Model predictions (redsolid histogram) to results including first new physics effects induced by a non-zero $\bar{c}_{W}=0.1$ parameter (black-solid line) and second by a non-zero $\bar{c}_{H W}=0.1$ parameter (blue-dotted line). While the Standard Model expectation steeply falls for invariant mass larger than $500 \mathrm{GeV}-600 \mathrm{GeV}$, beyond the Standard Model results exhibit a tail extending up to much larger $m_{V h}$ values around the $\mathrm{TeV}$ scale. New operators indeed contribute to this process with different kinematics, favoring configurations with larger four-momentum. This example therefore demonstrates the powerful usage of such an observable for unraveling new physics in the Higgs sector.

\subsection{Di-Higgs production in vector boson fusion}

Recently, the interest for di-Higgs production at the LHC, running at a center-of-mass energy of $14 \mathrm{TeV}$, has importantly increased. This process indeed allows to get a first grip 


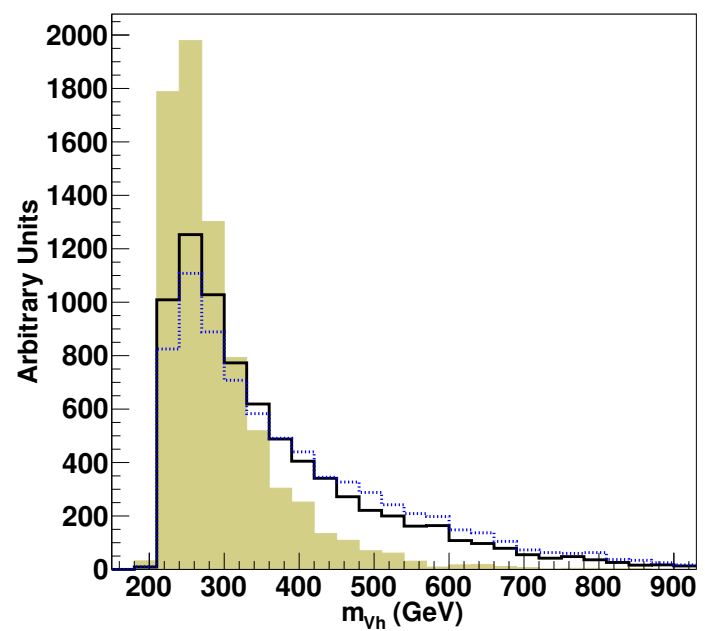

Figure 5. Invariant-mass $m_{V h}$ distribution of a two-body system comprised of a Higgs boson and a gauge boson for LHC collisions at a center-of-mass energy of $14 \mathrm{TeV}$. We show results for the Standard Model (red-solid histogram) to which we superimpose predictions computed when $\bar{c}_{H W}=0.1$ (blue-dotted line) and $\bar{c}_{W}=0.1$ (black-solid line) couplings are allowed.

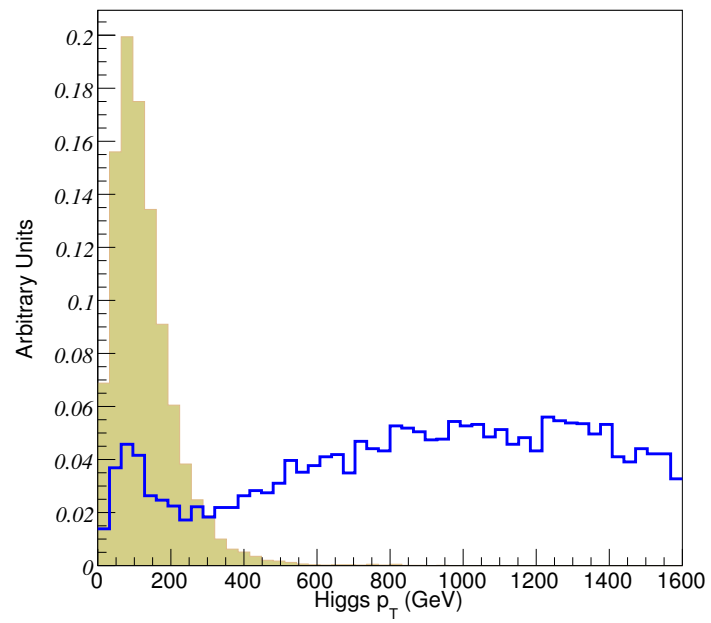

Figure 6. Transverse-momentum spectrum of both Higgs bosons of a $h h$ pair produced by vector boson fusion, the results including hence two entries for each event. The green-solid histogram depicts the SM predictions, while the blue-solid line corresponds to the addition of new physics effects modeled by $\bar{c}_{W}=0.05$.

on the triple-Higgs interaction strength. In the following, we show as an example di-Higgs production in the vector boson fusion mode,

$$
p p \rightarrow h h j j,
$$


where both jets $j$ are forward jets. Several of the effective operators of section 2.1 can affect such a process and we find that the distribution of the Higgs transverse momentum $p_{T}$ provides information allowing to probe such effects. This is illustrated on figure 6 where we represent the $p_{T}$ spectrum of both Higgs bosons, including hence one entry for each Higgs boson in the histograms. We compare the Standard Model expectation (green-solid histogram) to new physics results arising from $\bar{c}_{W}=0.05$ (blue-solid line).

We demonstrate in this way how the operator $\mathcal{O}_{W}$ (and the associated Wilson coefficient $\bar{c}_{W}$ ) favors final state configurations with boosted Higgs bosons. This behavior is even more pronounced than the one observed in section 4.2.2 when the Higgs boson is produced in association with a massive gauge boson. As a consequence, the operator $\mathcal{O}_{W}$ is likely to be investigated via new techniques dedicated to Higgs searches in boosted topologies, as presented for example in refs. [61, 158-160].

\subsection{Associated production of a Higgs and gauge boson from contact interac- tions with fermions}

As a last example of the strength of our implementation, we investigate, in this subsection, possible effects originating from effective operators involving one single Higgs field, one single gauge boson and a fermion-antifermion pair. More especially, we focus on the $\mathcal{O}_{H Q}$ operator of eq. (2.11) which contributes to the associated production of a Higgs boson $h$ together with a massive vector boson $V$ through a contact interaction,

$$
p p \rightarrow h V
$$

The kinematical properties due to new physics contributions are thus expected to be largely different from the Standard Model ones where the final state is produced via Higgs radiation from an off-shell gauge boson, as shown in eq. (4.10). Therefore, observables such as the transverse momentum of the Higgs boson or the invariant mass of the $V h$ system can be foreseen to play key roles in the detection of effects due to a non-vanishing $\bar{c}_{H Q}$ parameter. We have however found that this operator induces more striking modifications of the total production cross section, turning thus out to be more promising for searches for this type of physics beyond the Standard Model.

Considering Higgs boson production in association with a $Z$-boson, we restrict our analysis to a dileptonic $Z$-decay into an electron or muon pair, together with a Higgs boson decay into a pair of $b$-quarks,

$$
p p \rightarrow h Z \rightarrow(b \bar{b})\left(\ell^{+} \ell^{-}\right) .
$$

Simulating LHC collisions at a center-of-mass energy at $14 \mathrm{TeV}$, we impose that the four produced fermions have a transverse momentum larger than $25 \mathrm{GeV}$, a pseudorapidity satisfying $|\eta|>2.5$ and that the angular separation $\Delta R$ between any two objects is greater than 0.4. Normalizing the associated total production rate to the Standard Model one $\sigma_{S M}$, one fits the effects of the $\mathcal{O}_{H Q}$ operator as

$$
\kappa_{\bar{c}_{H Q}}=\frac{\sigma_{\bar{c}_{H Q}}}{\sigma_{S M}}=1.00-2.00 \bar{c}_{H Q}+863 \bar{c}_{H Q}^{2},
$$


where $\sigma_{\bar{c}_{H Q}}$ stands for the cross section as computed when including, in addition to the $\mathrm{SM}$ contributions, diagrams involving the $\mathcal{O}_{H Q}$ effective operator. This proves that any LHC measurement of this $\kappa_{\bar{c}_{H Q}}$ quantity that is accurate at the percent level (achievable with the high-luminosity run of the LHC [161]) would surpass the current bounds derived from LEP-I data at the Z-pole, the latter constraining the value of $\bar{c}_{H Q}$ to be of order $\mathcal{O}\left(10^{-3}\right)[162]$.

\section{Conclusions}

The Higgs discovery, together with the characterization of its properties and quantum numbers, sets the ground for the approach taken in this paper. With the absence of any evidence for new physics, we have followed the path of effective field theories to describe beyond the Standard Model effects in the Higgs sector. We have first formulated in extensive details the Higgs Effective Lagrangian that we have employed, limiting ourselves to dimension-six operators involving the Higgs and/or gauge bosons and adopting the basis and conventions of refs. [93, 97]. Next, we have implemented this Lagrangian in the framework of FeynRules, a MATHEMATica package interfaced to several sophisticated Monte Carlo tools. Our implementation allows to study, in particular, various differential distributions related to processes involving one or several Higgs bosons in the context of the LHC collisions. In the high-energy run of the LHC at a center-of-mass energy of $14 \mathrm{TeV}$, more statistics will be collected, resulting in a better understanding of the Higgs properties. In these perspectives, fits involving the Higgs signal strengths will rapidly fall short to exploit all data, while angular, invariant-mass as well as many other differential distributions will be able to directly probe possible non-standard Lorentz structures of the Higgs interactions. We believe that our implementation will hence allow to unveil physics beyond the Standard Model if related to the Higgs sector.

We have illustrated the strengths of our machinery by making use of the UFO interface of FEYNRUles to pass the full set of interaction vertices included in our Higgs Effective Lagrangian to the event generator MADGRAPH 5, without any restriction on the Lorentz structures or on the number of external legs allowed in the vertices. For the sake of the example, we have considered Higgs boson production via gluon fusion and investigated its decay modes to massive vector bosons, Higgs boson associated production with a weak boson, di-Higgs production as well as the effects of contact interactions among a fermionantifermion pair, a Higgs boson and a gauge boson. In all those processes, we have studied either total rates, or several differential distributions, or both, and shown how this has allowed us to investigate how dimension-six operators could manifest themselves in LHC processes. Finally, we have also briefly addressed the way to disentangle the source of a specific effect among the set of effective operators possibly giving rise to it.

\section{Acknowledgments}

The authors are grateful to Fabio Maltoni and Kentarou Mawatari for enlightening discussions during all the phases of this project. The work of VS has been supported by 
the Science Technology and Facilities Council (STFC) under grant number ST/J000477/1, while the work of AA and BF has received partial support from the Theorie-LHC France initiative of the CNRS/IN2P3.

Open Access. This article is distributed under the terms of the Creative Commons Attribution License (CC-BY 4.0), which permits any use, distribution and reproduction in any medium, provided the original author(s) and source are credited.

\section{References}

[1] ATLAS collaboration, Observation of a new particle in the search for the Standard Model Higgs boson with the ATLAS detector at the LHC, Phys. Lett. B 716 (2012) 1 [arXiv: 1207.7214] [INSPIRE].

[2] CMS collaboration, Observation of a new boson at a mass of $125 \mathrm{GeV}$ with the CMS experiment at the LHC, Phys. Lett. B 716 (2012) 30 [arXiv:1207.7235] [INSPIRE].

[3] P.W. Higgs, Broken Symmetries and the Masses of Gauge Bosons, Phys. Rev. Lett. 13 (1964) 508 [INSPIRE].

[4] P.W. Higgs, Spontaneous Symmetry Breakdown without Massless Bosons, Phys. Rev. 145 (1966) 1156 [INSPIRE].

[5] J.R. Dell'Aquila and C.A. Nelson, $P$ or $C P$ Determination by Sequential Decays: V1 V2 Modes With Decays Into $\bar{\ell}$ epton $(A) \ell(B)$ And/or $\bar{q}(A) q(B)$, Phys. Rev. D 33 (1986) 80 [INSPIRE].

[6] J.R. Dell'Aquila and C.A. Nelson, Distinguishing a Spin 0 Technipion and an Elementary

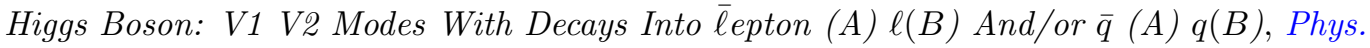
Rev. D 33 (1986) 93 [INSPIRE].

[7] J.R. Dell'Aquila and C.A. Nelson, Simple Tests for CP or P Violation by Sequential Decays: V1 V2 Modes With Decays Into $\bar{\ell}$ epton $(A) \ell(B)$ And/or $\bar{q}(A) q(B)$, Phys. Rev. D 33 (1986) 101 [INSPIRE].

[8] J.R. Dell'Aquila and C.A. Nelson, CP Determination for New Spin Zero Mesons by the $\bar{\tau} \tau$ Decay Mode, Nucl. Phys. B 320 (1989) 61 [INSPIRE].

[9] J.R. Dell'Aquila and C.A. Nelson, Usage of the $\bar{\tau} \tau$ or T Anti-t Decay Mode to Distinguish an Intermediate Mass Higgs Boson From a Technipion, Nucl. Phys. B 320 (1989) 86 [InSPIRE].

[10] S.Y. Choi, D.J. Miller, M.M. Muhlleitner and P.M. Zerwas, Identifying the Higgs spin and parity in decays to $Z$ pairs, Phys. Lett. B 553 (2003) 61 [hep-ph/0210077] [INSPIRE].

[11] K. Odagiri, On azimuthal spin correlations in Higgs plus jet events at LHC, JHEP 03 (2003) 009 [hep-ph/0212215] [INSPIRE].

[12] C.P. Buszello, I. Fleck, P. Marquard and J.J. van der Bij, Prospective analysis of spin- and $C P$-sensitive variables in $H \rightarrow Z Z \rightarrow l_{1}^{+} l_{1}^{-} l_{2}^{+} l_{2}^{-}$at the LHC, Eur. Phys. J. C 32 (2004) 209 [hep-ph/0212396] [INSPIRE].

[13] A. Djouadi, The Anatomy of electro-weak symmetry breaking. I: The Higgs boson in the standard model, Phys. Rept. 457 (2008) 1 [hep-ph/0503172] [INSPIRE].

[14] C.P. Buszello and P. Marquard, Determination of spin and CP of the Higgs boson from $W B F$, hep-ph/0603209 [inSPIRE]. 
[15] A. Bredenstein, A. Denner, S. Dittmaier and M.M. Weber, Precise predictions for the Higgs-boson decay $H \rightarrow W W / Z Z \rightarrow 4$ leptons, Phys. Rev. D 74 (2006) 013004 [hep-ph/0604011] [INSPIRE].

[16] P.S. Bhupal Dev, A. Djouadi, R.M. Godbole, M.M. Muhlleitner and S.D. Rindani, Determining the CP properties of the Higgs boson, Phys. Rev. Lett. 100 (2008) 051801 [arXiv: 0707.2878] [INSPIRE].

[17] R.M. Godbole, D.J. Miller and M.M. Muhlleitner, Aspects of CP-violation in the $H Z Z$ coupling at the LHC, JHEP 12 (2007) 031 [arXiv: 0708.0458] [INSPIRE].

[18] K. Hagiwara, Q. Li and K. Mawatari, Jet angular correlation in vector-boson fusion processes at hadron colliders, JHEP 07 (2009) 101 [arXiv: 0905.4314] [INSPIRE].

[19] Y. Gao, A.V. Gritsan, Z. Guo, K. Melnikov, M. Schulze et al., Spin determination of single-produced resonances at hadron colliders, Phys. Rev. D 81 (2010) 075022 [arXiv: 1001.3396] [INSPIRE].

[20] A. De Rujula, J. Lykken, M. Pierini, C. Rogan and M. Spiropulu, Higgs look-alikes at the LHC, Phys. Rev. D 82 (2010) 013003 [arXiv:1001.5300] [inSPIRE].

[21] M.R. Buckley and M.J. Ramsey-Musolf, Diagnosing Spin at the LHC via Vector Boson Fusion, JHEP 09 (2011) 094 [arXiv: 1008.5151] [INSPIRE].

[22] C. Englert, C. Hackstein and M. Spannowsky, Measuring spin and CP from semi-hadronic ZZ decays using jet substructure, Phys. Rev. D 82 (2010) 114024 [arXiv:1010.0676] [InSPIRE].

[23] U. De Sanctis, M. Fabbrichesi and A. Tonero, Telling the spin of the 'Higgs boson' at the LHC, Phys. Rev. D 84 (2011) 015013 [arXiv:1103.1973] [INSPIRE].

[24] V. Barger and P. Huang, Higgs boson finder and mass estimator: The Higgs boson to WW to leptons decay channel at the LHC, Phys. Rev. D 84 (2011) 093001 [arXiv:1107.4131] [INSPIRE].

[25] M.C. Kumar, P. Mathews, A.A. Pankov, N. Paver, V. Ravindran et al., Spin-analysis of s-channel diphoton resonances at the LHC, Phys. Rev. D 84 (2011) 115008 [arXiv:1108.3764] [INSPIRE].

[26] J. Ellis and D.S. Hwang, Does the 'Higgs' have Spin Zero?, JHEP 09 (2012) 071 [arXiv: 1202.6660] [INSPIRE].

[27] B. Coleppa, K. Kumar and H.E. Logan, Can the $126 \mathrm{GeV}$ boson be a pseudoscalar?, Phys. Rev. D 86 (2012) 075022 [arXiv: 1208.2692] [InSPIRE].

[28] S. Bolognesi, Y. Gao, A.V. Gritsan, K. Melnikov, M. Schulze et al., On the spin and parity of a single-produced resonance at the LHC, Phys. Rev. D 86 (2012) 095031 [arXiv:1208.4018] [INSPIRE].

[29] R. Boughezal, T.J. LeCompte and F. Petriello, Single-variable asymmetries for measuring the 'Higgs' boson spin and CP properties, arXiv:1208.4311 [INSPIRE].

[30] D. Stolarski and R. Vega-Morales, Directly Measuring the Tensor Structure of the Scalar Coupling to Gauge Bosons, Phys. Rev. D 86 (2012) 117504 [arXiv:1208.4840] [InSPIRE].

[31] J. Ellis, D.S. Hwang, V. Sanz and T. You, A Fast Track towards the 'Higgs' Spin and Parity, JHEP 11 (2012) 134 [arXiv:1208.6002] [INSPIRE].

[32] A. Alves, Is the New Resonance Spin 0 or 2? Taking a Step Forward in the Higgs Boson Discovery, Phys. Rev. D 86 (2012) 113010 [arXiv:1209.1037] [INSPIRE]. 
[33] P. Cea, Comment on the evidence of the Higgs boson at LHC, arXiv:1209.3106 [INSPIRE].

[34] S.Y. Choi, M.M. Muhlleitner and P.M. Zerwas, Theoretical Basis of Higgs-Spin Analysis in $H \rightarrow \gamma \gamma$ and $Z \gamma$ Decays, Phys. Lett. B 718 (2013) 1031 [arXiv:1209.5268] [INSPIRE].

[35] P. Avery, D. Bourilkov, M. Chen, T. Cheng, A. Drozdetskiy et al., Precision studies of the Higgs boson decay channel $H \rightarrow Z Z \rightarrow 4$ l with MEKD, Phys. Rev. D 87 (2013) 055006 [arXiv: 1210.0896] [INSPIRE].

[36] C.-Q. Geng, D. Huang, Y. Tang and Y.-L. Wu, Note on 125 GeV Spin-2 particle, Phys. Lett. B 719 (2013) 164 [arXiv:1210.5103] [INSPIRE].

[37] J. Ellis, R. Fok, D.S. Hwang, V. Sanz and T. You, Distinguishing 'Higgs' spin hypotheses using $\gamma \gamma$ and $W W^{*}$ decays, Eur. Phys. J. C 73 (2013) 2488 [arXiv:1210.5229] [InSPIRE].

[38] A. Freitas and P. Schwaller, Higgs CP Properties From Early LHC Data, Phys. Rev. D 87 (2013) 055014 [arXiv:1211.1980] [INSPIRE].

[39] J.R. Andersen, C. Englert and M. Spannowsky, Extracting precise Higgs couplings by using the matrix element method, Phys. Rev. D 87 (2013) 015019 [arXiv:1211.3011] [InSPIRE].

[40] J. Ellis, V. Sanz and T. You, Prima Facie Evidence against Spin-Two Higgs Impostors, Phys. Lett. B 726 (2013) 244 [arXiv:1211.3068] [INSPIRE].

[41] J. Frank, M. Rauch and D. Zeppenfeld, Spin-2 Resonances in Vector-Boson-Fusion Processes at NLO QCD, Phys. Rev. D 87 (2013) 055020 [arXiv:1211.3658] [INSPIRE].

[42] C. Englert, D. Goncalves-Netto, K. Mawatari and T. Plehn, Higgs Quantum Numbers in Weak Boson Fusion, JHEP 01 (2013) 148 [arXiv:1212.0843] [INSPIRE].

[43] C. Bernaciak, M.S.A. Buschmann, A. Butter and T. Plehn, Fox-Wolfram Moments in Higgs Physics, Phys. Rev. D 87 (2013) 073014 [arXiv:1212.4436] [InSPIRE].

[44] A. Djouadi, R.M. Godbole, B. Mellado and K. Mohan, Probing the spin-parity of the Higgs boson via jet kinematics in vector boson fusion, Phys. Lett. B 723 (2013) 307 [arXiv: 1301.4965] [INSPIRE].

[45] T. Modak, D. Sahoo, R. Sinha and H.-Y. Cheng, Inferring the nature of the boson at 125-126 GeV, arXiv:1301.5404 [INSPIRE].

[46] P. Artoisenet, P. de Aquino, F. Demartin, R. Frederix, S. Frixione et al., A framework for Higgs characterisation, JHEP 11 (2013) 043 [arXiv:1306.6464] [INSPIRE].

[47] CDF Collaboration, D0 collaboration, T. Aaltonen et al., Higgs Boson Studies at the Tevatron, Phys. Rev. D 88 (2013) 052014 [arXiv: 1303.6346] [INSPIRE].

[48] ATLAS collaboration, Combined coupling measurements of the Higgs-like boson with the ATLAS detector using up to $25 \mathrm{fb}^{-1}$ of proton-proton collision data, ATLAS-CONF-2013-034.

[49] CMS collaboration, Study of the Mass and Spin-Parity of the Higgs Boson Candidate Via Its Decays to Z Boson Pairs, Phys. Rev. Lett. 110 (2013) 081803 [arXiv:1212.6639] [INSPIRE].

[50] D. Carmi, A. Falkowski, E. Kuflik and T. Volansky, Interpreting LHC Higgs Results from Natural New Physics Perspective, JHEP 07 (2012) 136 [arXiv:1202.3144] [INSPIRE].

[51] A. Azatov, R. Contino and J. Galloway, Model-Independent Bounds on a Light Higgs, JHEP 04 (2012) 127 [Erratum ibid. 1304 (2013) 140] [arXiv: 1202.3415] [INSPIRE]. 
[52] J.R. Espinosa, C. Grojean, M. Muhlleitner and M. Trott, Fingerprinting Higgs Suspects at the LHC, JHEP 05 (2012) 097 [arXiv: 1202.3697] [INSPIRE].

[53] P.P. Giardino, K. Kannike, M. Raidal and A. Strumia, Reconstructing Higgs boson properties from the LHC and Tevatron data, JHEP 06 (2012) 117 [arXiv:1203.4254] [INSPIRE].

[54] T. Li, X. Wan, Y.-k. Wang and S.-h. Zhu, Constraints on the Universal Varying Yukawa Couplings: from SM-like to Fermiophobic, JHEP 09 (2012) 086 [arXiv:1203.5083] [INSPIRE].

[55] M. Rauch, Determination of Higgs-boson couplings (SFitter), arXiv:1203.6826 [INSPIRE].

[56] J. Ellis and T. You, Global Analysis of Experimental Constraints on a Possible Higgs-Like Particle with Mass $125 \mathrm{GeV}$, JHEP 06 (2012) 140 [arXiv:1204.0464] [INSPIRE].

[57] A. Azatov, R. Contino, D. Del Re, J. Galloway, M. Grassi et al., Determining Higgs couplings with a model-independent analysis of $h$-¿gamma gamma, JHEP 06 (2012) 134 [arXiv: 1204 .4817] [INSPIRE].

[58] M. Klute, R. Lafaye, T. Plehn, M. Rauch and D. Zerwas, Measuring Higgs Couplings from LHC Data, Phys. Rev. Lett. 109 (2012) 101801 [arXiv:1205.2699] [INSPIRE].

[59] J.R. Espinosa, M. Muhlleitner, C. Grojean and M. Trott, Probing for Invisible Higgs Decays with Global Fits, JHEP 09 (2012) 126 [arXiv:1205.6790] [INSPIRE].

[60] D. Carmi, A. Falkowski, E. Kuflik and T. Volansky, Interpreting the Higgs, Frascati Phys. Ser. 57 (2013) 315 [arXiv: 1206.4201] [INSPIRE].

[61] M.J. Dolan, C. Englert and M. Spannowsky, Higgs self-coupling measurements at the LHC, JHEP 10 (2012) 112 [arXiv:1206.5001] [INSPIRE].

[62] J. Chang, K. Cheung, P.-Y. Tseng and T.-C. Yuan, Distinguishing Various Models of the 125 GeV Boson in Vector Boson Fusion, JHEP 12 (2012) 058 [arXiv: 1206.5853] [INSPIRE].

[63] S. Chang, C.A. Newby, N. Raj and C. Wanotayaroj, Revisiting Theories with Enhanced Higgs Couplings to Weak Gauge Bosons, Phys. Rev. D 86 (2012) 095015 [arXiv:1207.0493] [INSPIRE].

[64] I. Low, J. Lykken and G. Shaughnessy, Have We Observed the Higgs (Imposter)?, Phys. Rev. D 86 (2012) 093012 [arXiv:1207.1093] [INSPIRE].

[65] P.P. Giardino, K. Kannike, M. Raidal and A. Strumia, Is the resonance at $125 \mathrm{GeV}$ the Higgs boson?, Phys. Lett. B 718 (2012) 469 [arXiv:1207.1347] [INSPIRE].

[66] M. Montull and F. Riva, Higgs discovery: the beginning or the end of natural EWSB?, JHEP 11 (2012) 018 [arXiv:1207.1716] [INSPIRE].

[67] J.R. Espinosa, C. Grojean, M. Muhlleitner and M. Trott, First Glimpses at Higgs' face, JHEP 12 (2012) 045 [arXiv:1207.1717] [InSPIRE].

[68] D. Carmi, A. Falkowski, E. Kuflik, T. Volansky and J. Zupan, Higgs After the Discovery: A Status Report, JHEP 10 (2012) 196 [arXiv:1207.1718] [INSPIRE].

[69] S. Banerjee, S. Mukhopadhyay and B. Mukhopadhyaya, New Higgs interactions and recent data from the LHC and the Tevatron, JHEP 10 (2012) 062 [arXiv:1207.3588] [INSPIRE].

[70] F. Bonnet, T. Ota, M. Rauch and W. Winter, Interpretation of precision tests in the Higgs sector in terms of physics beyond the Standard Model, Phys. Rev. D 86 (2012) 093014 [arXiv: 1207.4599] [INSPIRE]. 
[71] T. Plehn and M. Rauch, Higgs Couplings after the Discovery, Europhys. Lett. 100 (2012) 11002 [arXiv: 1207.6108 ] [INSPIRE].

[72] J.R. Espinosa, C. Grojean, V. Sanz and M. Trott, NSUSY fits, JHEP 12 (2012) 077 [arXiv: 1207.7355] [INSPIRE].

[73] A. Djouadi, Precision Higgs coupling measurements at the LHC through ratios of production cross sections, Eur. Phys. J. C 73 (2013) 2498 [arXiv:1208.3436] [INSPIRE].

[74] J. Chang, K. Cheung, P.-Y. Tseng and T.-C. Yuan, Various Models Mimicking the SM Higgs Boson, Int. J. Mod. Phys. A 27 (2012) 1230030 [arXiv:1211.6823] [INSPIRE].

[75] G. Bélanger, B. Dumont, U. Ellwanger, J.F. Gunion and S. Kraml, Higgs Couplings at the End of 2012, JHEP 02 (2013) 053 [arXiv: 1212.5244] [INSPIRE].

[76] A. Djouadi and G. Moreau, The couplings of the Higgs boson and its CP properties from fits of the signal strengths and their ratios at the 7+8 TeV LHC, arXiv:1303.6591 [INSPIRE].

[77] G. Bélanger, B. Dumont, U. Ellwanger, J.F. Gunion and S. Kraml, Global fit to Higgs signal strengths and couplings and implications for extended Higgs sectors, Phys. Rev. D 88 (2013) 075008 [arXiv: 1306 .2941] [inSPIRE].

[78] G. Altarelli, The Higgs: so simple yet so unnatural, Phys. Scripta T 158 (2013) 014011 [arXiv: 1308.0545] [INSPIRE].

[79] S. Banerjee, S. Mukhopadhyay and B. Mukhopadhyaya, Higher dimensional operators and LHC Higgs data: the role of modified kinematics, Phys. Rev. D 89 (2014) 053010 [arXiv: 1308.4860] [INSPIRE].

[80] https://twiki.cern.ch/twiki/bin/view/AtlasPublic.

[81] https://twiki.cern.ch/twiki/bin/view/CMSPublic/PhysicsResults.

[82] CMS collaboration, A search for anomalous production of events with three or more leptons using 19.5/fb of $\sqrt{s}=8$ TeV LHC data, CMS-PAS-SUS-13-002.

[83] J. D'Hondt, K. De Causmaecker, B. Fuks, A. Mariotti, K. Mawatari et al., Multilepton signals of gauge mediated supersymmetry breaking at the LHC, Phys. Lett. B 731 (2014) 7 [arXiv: 1310.0018] [INSPIRE].

[84] C.J.C. Burges and H.J. Schnitzer, Virtual Effects of Excited Quarks as Probes of a Possible New Hadronic Mass Scale, Nucl. Phys. B 228 (1983) 464 [INSPIRE].

[85] C.N. Leung, S.T. Love and S. Rao, Low-Energy Manifestations of a New Interaction Scale: Operator Analysis, Z. Phys. C 31 (1986) 433 [INSPIRE].

[86] W. Buchmüller and D. Wyler, Effective Lagrangian Analysis of New Interactions and Flavor Conservation, Nucl. Phys. B 268 (1986) 621 [INSPIRE].

[87] A. De Rujula, M.B. Gavela, P. Hernández and E. Masso, The Selfcouplings of vector bosons: Does LEP-1 obviate LEP-2?, Nucl. Phys. B 384 (1992) 3 [inSPIRE].

[88] K. Hagiwara, S. Ishihara, R. Szalapski and D. Zeppenfeld, Low-energy constraints on electroweak three gauge boson couplings, Phys. Lett. B 283 (1992) 353 [InSPIRE].

[89] K. Hagiwara, S. Ishihara, R. Szalapski and D. Zeppenfeld, Low-energy effects of new interactions in the electroweak boson sector, Phys. Rev. D 48 (1993) 2182 [INSPIRE].

[90] K. Hagiwara, R. Szalapski and D. Zeppenfeld, Anomalous Higgs boson production and decay, Phys. Lett. B 318 (1993) 155 [hep-ph/9308347] [INSPIRE]. 
[91] M.C. Gonzalez-Garcia, Anomalous Higgs couplings, Int. J. Mod. Phys. A 14 (1999) 3121 [hep-ph/9902321] [INSPIRE].

[92] O.J.P. Eboli, M.C. Gonzalez-Garcia, S. . Lietti and S.F. Novaes, Probing intermediate mass Higgs interactions at the CERN Large Hadron Collider, Phys. Lett. B 478 (2000) 199 [hep-ph/0001030] [INSPIRE].

[93] G.F. Giudice, C. Grojean, A. Pomarol and R. Rattazzi, The Strongly-Interacting Light Higgs, JHEP 06 (2007) 045 [hep-ph/0703164] [INSPIRE].

[94] B. Grzadkowski, M. Iskrzynski, M. Misiak and J. Rosiek, Dimension-Six Terms in the Standard Model Lagrangian, JHEP 10 (2010) 085 [arXiv:1008.4884] [INSPIRE].

[95] F. Bonnet, M.B. Gavela, T. Ota and W. Winter, Anomalous Higgs couplings at the LHC and their theoretical interpretation, Phys. Rev. D 85 (2012) 035016 [arXiv:1105.5140] [INSPIRE].

[96] S.S. Biswal, R.M. Godbole, B. Mellado and S. Raychaudhuri, Azimuthal Angle Probe of Anomalous HWW Couplings at a High Energy ep Collider, Phys. Rev. Lett. 109 (2012) 261801 [arXiv: 1203.6285] [INSPIRE].

[97] R. Contino, M. Ghezzi, C. Grojean, M. Muhlleitner and M. Spira, Effective Lagrangian for a light Higgs-like scalar, JHEP 07 (2013) 035 [arXiv: 1303.3876] [INSPIRE].

[98] J.S. Gainer, J. Lykken, K.T. Matchev, S. Mrenna and M. Park, Geolocating the Higgs Boson Candidate at the LHC, Phys. Rev. Lett. 111 (2013) 041801 [arXiv:1304.4936] [INSPIRE].

[99] M. Chen, T. Cheng, J.S. Gainer, A. Korytov, K.T. Matchev et al., The role of interference in unraveling the ZZ-couplings of the newly discovered boson at the LHC, Phys. Rev. D 89 (2014) 034002 [arXiv:1310.1397] [INSPIRE].

[100] T. Corbett, O.J.P. Eboli, J. Gonzalez-Fraile and M.C. Gonzalez-Garcia, Constraining anomalous Higgs interactions, Phys. Rev. D 86 (2012) 075013 [arXiv:1207.1344] [INSPIRE].

[101] E. Masso and V. Sanz, Limits on Anomalous Couplings of the Higgs to Electroweak Gauge Bosons from LEP and LHC, Phys. Rev. D 87 (2013) 033001 [arXiv:1211.1320] [InSPIRE].

[102] T. Corbett, O.J.P. Eboli, J. Gonzalez-Fraile and M.C. Gonzalez-Garcia, Robust Determination of the Higgs Couplings: Power to the Data, Phys. Rev. D 87 (2013) 015022 [arXiv: 1211.4580] [INSPIRE].

[103] T. Corbett, O.J.P. Éboli, J. Gonzalez-Fraile and M.C. Gonzalez-Garcia, Determining Triple Gauge Boson Couplings from Higgs Data, Phys. Rev. Lett. 111 (2013) 011801 [arXiv: 1304.1151] [INSPIRE].

[104] B. Dumont, S. Fichet and G. von Gersdorff, A Bayesian view of the Higgs sector with higher dimensional operators, JHEP 07 (2013) 065 [arXiv: 1304.3369] [INSPIRE].

[105] T. Corbett, O.J.P. Éboli, J. Gonzalez-Fraile and M.C. Gonzalez-Garcia, Robust determination of the scalar boson couplings, arXiv:1306.0006 [INSPIRE].

[106] N.D. Christensen and C. Duhr, FeynRules - Feynman rules made easy, Comput. Phys. Commun. 180 (2009) 1614 [arXiv:0806.4194] [InSPIRE].

[107] N.D. Christensen, C. Duhr, B. Fuks, J. Reuter and C. Speckner, Introducing an interface between WHIZARD and FeynRules, Eur. Phys. J. C 72 (2012) 1990 [arXiv:1010.3251] [INSPIRE].

[108] C. Duhr and B. Fuks, A superspace module for the FeynRules package, Comput. Phys. Commun. 182 (2011) 2404 [arXiv:1102.4191] [INSPIRE]. 
[109] B. Fuks, Beyond the Minimal Supersymmetric Standard Model: from theory to phenomenology, Int. J. Mod. Phys. A 27 (2012) 1230007 [arXiv:1202.4769] [InSPIRE].

[110] A. Alloul, J. D'Hondt, K. De Causmaecker, B. Fuks and M. Rausch de Traubenberg, Automated mass spectrum generation for new physics, Eur. Phys. J. C 73 (2013) 2325 [arXiv: 1301.5932] [INSPIRE].

[111] N.D. Christensen, P. de Aquino, N. Deutschmann, C. Duhr, B. Fuks et al., Simulating spin- $\frac{3}{2}$ particles at colliders, Eur. Phys. J. C 73 (2013) 2580 [arXiv:1308.1668] [inSPIRE].

[112] A. Alloul, N.D. Christensen, C. Degrande, C. Duhr and B. Fuks, New developments in FeynRules, arXiv:1309.7806 [INSPIRE].

[113] A. Alloul, N.D. Christensen, C. Degrande, C. Duhr and B. Fuks, FeynRules 2.0 - A complete toolbox for tree-level phenomenology, arXiv:1310.1921 [INSPIRE].

[114] N.D. Christensen, P. de Aquino, C. Degrande, C. Duhr, B. Fuks et al., A Comprehensive approach to new physics simulations, Eur. Phys. J. C 71 (2011) 1541 [arXiv:0906.2474] [INSPIRE].

[115] A. Pukhov, E. Boos, M. Dubinin, V. Edneral, V. Ilyin et al., CompHEP: A Package for evaluation of Feynman diagrams and integration over multiparticle phase space, hep-ph/9908288 [INSPIRE].

[116] CompHEP collaboration, E. Boos et al., CompHEP 4.4: Automatic computations from Lagrangians to events, Nucl. Instrum. Meth. A 534 (2004) 250 [hep-ph/0403113] [INSPIRE].

[117] A. Pukhov, CalcHEP 2.3: MSSM, structure functions, event generation, batchs and generation of matrix elements for other packages, hep-ph/0412191 [INSPIRE].

[118] A. Belyaev, N.D. Christensen and A. Pukhov, CalcHEP 3.4 for collider physics within and beyond the Standard Model, Comput. Phys. Commun. 184 (2013) 1729 [arXiv:1207.6082] [INSPIRE].

[119] T. Hahn and M. Pérez-Victoria, Automatized one loop calculations in four-dimensions and D-dimensions, Comput. Phys. Commun. 118 (1999) 153 [hep-ph/9807565] [INSPIRE].

[120] T. Hahn, Generating Feynman diagrams and amplitudes with FeynArts 3, Comput. Phys. Commun. 140 (2001) 418 [hep-ph/0012260] [INSPIRE].

[121] T. Hahn, A Mathematica interface for FormCalc-generated code, Comput. Phys. Commun. 178 (2008) 217 [hep-ph/0611273] [INSPIRE].

[122] T. Hahn, FormCalc 6, PoS (ACAT08) 121 [arXiv:0901.1528] [INSPIRE].

[123] S. Agrawal, T. Hahn and E. Mirabella, FormCalc 7, J. Phys. Conf. Ser. 368 (2012) 012054 [arXiv: 1112.0124] [INSPIRE].

[124] B. Chokoufe Nejad, T. Hahn, J.N. Lang and E. Mirabella, FormCalc 8: Better Algebra and Vectorization, arXiv:1310.0274 [INSPIRE].

[125] T. Stelzer and W.F. Long, Automatic generation of tree level helicity amplitudes, Comput. Phys. Commun. 81 (1994) 357 [hep-ph/9401258] [INSPIRE].

[126] F. Maltoni and T. Stelzer, MadEvent: Automatic event generation with MadGraph, JHEP 02 (2003) 027 [hep-ph/0208156] [INSPIRE].

[127] J. Alwall, P. Demin, S. de Visscher, R. Frederix, M. Herquet et al., MadGraph/MadEvent v4: The New Web Generation, JHEP 09 (2007) 028 [arXiv: 0706.2334] [INSPIRE]. 
[128] J. Alwall, P. Artoisenet, S. de Visscher, C. Duhr, R. Frederix et al., New Developments in MadGraph/MadEvent, AIP Conf. Proc. 1078 (2009) 84 [arXiv:0809.2410] [InSPIRE].

[129] J. Alwall, M. Herquet, F. Maltoni, O. Mattelaer and T. Stelzer, MadGraph 5 : Going Beyond, JHEP 06 (2011) 128 [arXiv:1106.0522] [INSPIRE].

[130] T. Gleisberg, S. Hoeche, F. Krauss, A. Schalicke, S. Schumann et al., SHERPA 1. alpha: A Proof of concept version, JHEP 02 (2004) 056 [hep-ph/0311263] [INSPIRE].

[131] T. Gleisberg, S. Hoeche, F. Krauss, M. Schonherr, S. Schumann et al., Event generation with SHERPA 1.1, JHEP 02 (2009) 007 [arXiv: 0811.4622] [INSPIRE].

[132] M. Moretti, T. Ohl and J. Reuter, O'Mega: An Optimizing matrix element generator, hep-ph/0102195 [INSPIRE].

[133] W. Kilian, T. Ohl and J. Reuter, WHIZARD: Simulating Multi-Particle Processes at LHC and ILC, Eur. Phys. J. C 71 (2011) 1742 [arXiv:0708.4233] [INSPIRE].

[134] C. Degrande, C. Duhr, B. Fuks, D. Grellscheid, O. Mattelaer et al., UFO - The Universal FeynRules Output, Comput. Phys. Commun. 183 (2012) 1201 [arXiv:1108.2040] [inSPIRE].

[135] P. de Aquino, W. Link, F. Maltoni, O. Mattelaer and T. Stelzer, ALOHA: Automatic Libraries Of Helicity Amplitudes for Feynman Diagram Computations, Comput. Phys. Commun. 183 (2012) 2254 [arXiv:1108.2041] [INSPIRE].

[136] G. Cullen, N. Greiner, G. Heinrich, G. Luisoni, P. Mastrolia et al., Automated One-Loop Calculations with GoSam, Eur. Phys. J. C 72 (2012) 1889 [arXiv:1111.2034] [INSPIRE].

[137] G. Cullen, N. Greiner, G. Heinrich, G. Luisoni, P. Mastrolia et al., GoSam: A Program for Automated One-Loop Calculations, J. Phys. Conf. Ser. 368 (2012) 012056 [arXiv:1111.6534] [INSPIRE].

[138] M. Bahr, S. Gieseke, M.A. Gigg, D. Grellscheid, K. Hamilton et al., HERWIG++ Physics and Manual, Eur. Phys. J. C 58 (2008) 639 [arXiv:0803.0883] [InSPIRE].

[139] K. Arnold, L. d'Errico, S. Gieseke, D. Grellscheid, K. Hamilton et al., HERWIG++ 2.6 Release Note, arXiv:1205.4902 [INSPIRE].

[140] E. Conte, B. Fuks and G. Serret, MadAnalysis 5, A User-Friendly Framework for Collider Phenomenology, Comput. Phys. Commun. 184 (2013) 222 [arXiv:1206.1599] [INSPIRE].

[141] E. Conte and B. Fuks, MadAnalysis 5: status and new developments, arXiv:1309.7831 [INSPIRE].

[142] M. Baak, M. Goebel, J. Haller, A. Hoecker, D. Kennedy et al., The Electroweak Fit of the Standard Model after the Discovery of a New Boson at the LHC, Eur. Phys. J. C 72 (2012) 2205 [arXiv: 1209. 2716] [INSPIRE].

[143] V.D. Barger, G. Bhattacharya, T. Han and B.A. Kniehl, Intermediate mass Higgs boson at hadron supercolliders, Phys. Rev. D 43 (1991) 779 [INSPIRE].

[144] M. Dittmar and H.K. Dreiner, How to find a Higgs boson with a mass between 155-GeV 180-GeV at the LHC, Phys. Rev. D 55 (1997) 167 [hep-ph/9608317] [INSPIRE].

[145] M. Dittmar and H. Dreiner, LHC Higgs Search with $l^{+} \nu l^{-} \bar{\nu}$ Final States, CMS-NOTE-1997-083.

[146] J. Frank, M. Rauch and D. Zeppenfeld, Higgs Spin Determination in the $W W$ channel and beyond, arXiv: 1305.1883 [INSPIRE]. 
[147] CMS collaboration, Combination of standard model Higgs boson searches and measurements of the properties of the new boson with a mass near $125 \mathrm{GeV}$, CMS-PAS-HIG-13-005.

[148] ATLAS collaboration, Measurements of Higgs boson production and couplings in diboson final states with the ATLAS detector at the LHC, Phys. Lett. B 726 (2013) 88 [arXiv: 1307.1427] [INSPIRE].

[149] A. Djouadi, J. Kalinowski and M. Spira, HDECAY: A Program for Higgs boson decays in the standard model and its supersymmetric extension, Comput. Phys. Commun. 108 (1998) 56 [hep-ph/9704448] [INSPIRE].

[150] J.M. Butterworth, A. Arbey, L. Basso, S. Belov, A. Bharucha et al., The Tools and Monte Carlo working group Summary Report, arXiv:1003.1643 [INSPIRE].

[151] V.D. Barger, K.-m. Cheung, A. Djouadi, B.A. Kniehl and P.M. Zerwas, Higgs bosons: Intermediate mass range at $e^{+} e^{-}$colliders, Phys. Rev. D 49 (1994) 79 [hep-ph/9306270] [INSPIRE].

[152] C. Englert, D. Goncalves, G. Nail and M. Spannowsky, The shape of spins, Phys. Rev. D 88 (2013) 013016 [arXiv: 1304.0033] [INSPIRE].

[153] J. Ellis, V. Sanz and T. You, Associated Production Evidence against Higgs Impostors and Anomalous Couplings, Eur. Phys. J. C 73 (2013) 2507 [arXiv:1303.0208] [InSPIRE].

[154] R. Godbole, D.J. Miller, K. Mohan and C.D. White, Boosting Higgs CP properties via VH Production at the Large Hadron Collider, Phys. Lett. B 730 (2014) 275 [arXiv:1306.2573] [INSPIRE].

[155] G. Isidori and M. Trott, Higgs form factors in Associated Production, JHEP 02 (2014) 082 [arXiv: 1307.4051] [INSPIRE].

[156] M.L. Mangano and J. Rojo, Cross Section Ratios between different CM energies at the LHC: opportunities for precision measurements and BSM sensitivity, JHEP 08 (2012) 010 [arXiv:1206.3557] [INSPIRE].

[157] F. Boudjema, G. Cacciapaglia, K. Cranmer, G. Dissertori, A. Deandrea et al., On the presentation of the LHC Higgs Results, arXiv:1307.5865 [INSPIRE].

[158] M.J. Dolan, C. Englert and M. Spannowsky, New Physics in LHC Higgs boson pair production, Phys. Rev. D 87 (2013) 055002 [arXiv:1210.8166] [INSPIRE].

[159] M. Gouzevitch, A. Oliveira, J. Rojo, R. Rosenfeld, G.P. Salam et al., Scale-invariant resonance tagging in multijet events and new physics in Higgs pair production, JHEP $\mathbf{0 7}$ (2013) 148 [arXiv:1303.6636] [INSPIRE].

[160] M.J. Dolan, C. Englert, N. Greiner and M. Spannowsky, Further on up the road: hhjj production at the LHC, Phys. Rev. Lett. 112 (2014) 101802 [arXiv:1310.1084] [InSPIRE].

[161] J.M. Butterworth, A.R. Davison, M. Rubin and G.P. Salam, Jet substructure as a new Higgs search channel at the LHC, arXiv:0810.0409 [INSPIRE].

[162] A. Pomarol and F. Riva, Towards the Ultimate SM Fit to Close in on Higgs Physics, JHEP 01 (2014) 151 [arXiv: 1308.2803] [inSPIRE]. 